\title{
Der Schematismus reiner Verstandesbegriffe bei Kant, seine Transformation im südwestdeutschen Neukantianismus und in der späteren kantianisierenden Transzendentalphilosophie: eine problemgeschichtliche Betrachtung
}

[O Esquematismo dos Conceitos puros do Entendimento em Kant, a sua Transformação no neo-kantismo do Sudoeste alemão e na Kantianização da Filosofia Transcendental Tardia: uma Consideração histórica do Problema]

\section{Christian Krijnen}

Zusammenfassung: Sinn und Geltung von Kants Schematismuslehre sind bis heute umstritten. Im Neukantianismus und in der Transzendentalphilosophie der Nachkriegszeit kommt es diesbezüglich zur einer radikalen Transformation von Kants Schematismus reiner Verstandesbegriffe. Kants Heterogenitätsthese soll überwunden werden durch eine Rückbindung der Heterogenität an eine fundierende Einheit, die die Geltungsstruktur der Erkenntnis selbst ist. Die Schematisierungsleistung der Spontaneität des Denkens wird festgehalten, Kants apperzeptionstheoretische Pointierung und die mit ihr einhergehende Äußerlichkeit von Gegebenem und Bestimmung des Gegebenen in einer geltungsnoematischen Konstitutions- und Regulationsordnung aufgehoben. Der positive Sinn von Kants Schematismuslehre erweist sich, ob bei den diskutierten Neukantianern oder der späteren Transzendentalphilosophie als Methodologie.

Schlüsselwörter: Schematismus. Spontanität. Rezeptivität. Heterogenität. Dualismus. Methodologie. geltungsnoematische Struktur. Neukantianismus. Transzendentalphilosophie.

Resumo: O sentido e a validade da doutrina kantiana do esquematismo são controversos até hoje. A esse respeito, há uma transformação radical do esquematismo kantiano dos conceitos puros do entendimento no neokantismo e na filosofia transcendental do período pós-guerra. A tese de Kant da heterogeneidade deve ser superada por meio de uma reconexão da heterogeneidade a uma unidade fundamental, a qual é a própria estrutura de validade do conhecimento. É mantida a capacidade de esquematização da espontaneidade do pensamento e são revogadas a ênfase de Kant na teoria da apercepção e a exterioridade do dado a qual o acompanha e a determinação do dado em uma ordem constitucional e regulatória válido-noemática. O sentido positivo da doutrina de Kant do esquematismo prova ser uma metodologia, seja nos neokantianos discutidos ou na filosofia transcendental posterior.

Palavras-chave: Esquematismo. Espontaneidade. Receptividade. Heterogeneidade. Dualismo. Metodologia. Estrutura noemática de validade. Neo-kantianismo. Filosofia transcendental.

${ }^{*}$ Doctor philosophiae (Dr.). VU University Amsterdam, Netherlands. Doctor philosophiae habilitatus (Dr. phil. habil.). University of Siegen, Germany. Associate Professor at VU University. E-mail: c.h.krijnen@vu.nl. https://vu-nl.academia.edu/ChristianKrijnen. 
In zwei neueren Dissertationen zu Kant spielt ein Problem eine Hauptrolle ${ }^{1}$, das in der Auseinandersetzung mit Kant immer schon eine bedeutsame Rolle gespielt hat und gerade durch Heideggers $(1962$; 1991) Kant-Deutung $\mathrm{zu}$ einer Schicksalsfrage hochstilisiert wurde: das Schematismusproblem. Die Breite der Auslegungsversuche ist erstaunlich, bedenkt man, daß Kant selbst das Schematismuskapitel als „eins der wichtigsten" Teile der Kritik der reinen Vernunft bezeichnet hatte (Refl. 6359, AA XVIII; vgl. Prol. § 34, AA IV, 316). Die Einschätzungen reichen von dessen Entbehrlichkeit angesichts der transzendentalen Deduktion reiner Verstandesbegriffe bis hin zur ursprünglichen
Begründung ebendieser Deduktion..$^{2}$

In der Diskussion fällt auf, daß die systematischen Versuche der Transzendentalphilosophie des südwestdeutschen Neukantianismus und von ihnen stark beeinflußten Denkern der Nachkriegszeit (wie Hans Wagner und Werner Flach), Kants These von der Heterogenität der Erkenntnisquellen des Verstandes und der Sinnlichkeit durch eine fundierende Einheit $\mathrm{zu}$ relativieren, nicht eigens diskutiert werden. $3^{3} \mathrm{He}$ gels Versuch, Kants ,dualistische' Konzeption durch eine spekulative Selbstvermittlungsstruktur des Begriffs zu überwinden, kommt freilich ebenfalls nicht als systematisches Konkurrenzangebot ernsthaft zur Sprache. Indes

\footnotetext{
${ }^{1}$ Vgl. Birrer (2017) und Bunte (2016). Beide legen auch den Forschungsstand dar (Birrer 2017, 11 ff.; Bunte 2016, 51 ff.). Vgl. zur Rezeption etwa auch: Allison (2004, 202 ff.); Düsing (1995, 47 ff.); Höffe (2003, 152 ff.).

${ }^{2}$ Vgl. vor diesem Hintergrund auch den Auslegungsversuch von Caimi (2015). Für Birrer (2017, 5 ff.) handelt es sich bei Caimis Deutung freilich um eine „revisionistische Lesart“, durch die die Sinnlichkeit im heterogenen Gefüge von Sinnlichkeit und Verstand marginalisiert wird; Birrer will ihr eine fortwährende Neubestimmung des Verhältnisses im Zuge des Kantischen Argumentationsgangs unter Beibehaltung der grundsätzlichen Eigenständigkeit der beiden Glieder entgegensetzen.

${ }^{3}$ Bunte (2017) bildet da eher die Ausnahme. Birrer, der wie Bunte nicht bloß historisch, sondern auch systematisch am Schematismusproblem interessiert ist, geht nicht auf die angedeutete Tradition der Transzendentalphilosophie ein. Er bezieht sich auf den frühen Neukantianer Riehl und vor allem auf die Marburger Neukantianer Cohen und Cassirer (Birrer 2017, 12 ff.); die Transzendentalphilosophie Wagners und Flachs, die sich gerade auch in Diskussion mit Kant entfaltet, spielt in seiner Darstellung der sog. „gegenwärtigen Kantforschung“ keine Rolle (Birrer (2017, 19 ff.). Er deutet die Pointe der neukantianischen Autoren so, daß sich ein „intellektueller Sinn begrifflicher Notwendigkeit bereits in den sinnlich anschaulichen Vorstellungen aufweisen lasse“ müsse. Indes wird die vorliegende Studie u. a. zeigen, daß für die diskutierte Tradition der Transzendentalphilosophie ebendiese von Birrer ins Spiel gebrachte Entgegensetzung von Anschauung und Begriff die ursprüngliche Zusammengehörigkeit der Momente der kognitiven Relation schon übersprungen hat. In dieser systematischen Hinsicht scheint mir die Arbeit von Bunte (2016) tiefschürfender. Entsprechend wird ihm Kants Begriff des Dinges an sich zum letzten Problem (Bunte 2016, Kap. 3.3). Nachdem Bunte sich bemüht hat, dessen Denkmöglichkeit darzulegen (2016, Kap. 3.3.1), erweist sich ihm die Erfahrungsmöglichkeit des Dinges an sich (2016, Kap. 3.3.2) als eine „Restschwierigkeit“ (2016, 303), genauer: als ein durch Kants Transzendentalphilosophie „unlösbares“ (2016, 310) Problem. Somit kommt es zu einer eigentümlichen Spannung zwischen dem von Bunte in seinem Buch mit großem Notwendigkeitspathos Vorgetragenen und der besagten Restschwierigkeit, mit der seine Untersuchung endet. Offenbar sind es gerade den Stämmedualismus betreffende Voraussetzungen Kants, die einer eingehenderen (,kritischen') Betrachtung bedürfen.
} 
fällt dem Schematismus sowohl in der kantianisierenden Transzendentalphilosophie als auch in Hegels spekulativem Idealismus nicht mehr die, wichtige' Rolle zu, die Kant ihm einst zugedacht hatte. Vielmehr sehen beide die Lösung des Schematismusproblems Kants darin, es gar nicht erst entstehen zu lassen.

Im folgenden soll dies mit Blick auf die kantianisierende Transzendentalphilosophie dargelegt werden. Meine These ist, daß die kantianisierende Transzendentalphilosophie tatsächlich einen wunden Punkt der Transzendentalphilosophie Kants abmildert. $\mathrm{Ob}$ sie damit auch der Hegelischen Herausforderung des Begriffs in gleichem Maße Herr wird, kann hier allerdings nicht weiter untersucht werden. Jedenfalls bedarf die Diskussion über die sachliche Relevanz von Kants Schematismustheorem Perspektiven, die die gängigen Deutungsschemata übersteigen.

\section{Das Problem des Schematismus rei- ner Verstandesbegriffe}

Unbestritten ist jedenfalls, daß Kants Schematismuskapitel ein Vermittlungsproblem traktiert, das sich aus der Architektonik von Kants Erkenntnislehre ergibt, und zwar dergestalt, daß die von Kant in Anschlag gebrachte Heterogenität von Anschauung (repraesentatio singularis, Einzelvorstellung) und Begriff (repraesentatio generalis, Allgemeinvorstellung) zwecks konkreter Gegenstandsbestimmung ein Verhältnis der Bezogenheit der heterogenen Elemente fordert. So gesehen betrifft das Schema ganz allgemein das Verhältnis eines unbestimmten Inhalts der Erkenntnis zu seiner bestimmenden Form (und nicht die klassenlogische ,Subsumtion' des Besonderen unter das Allgemeine): das eine muß mit dem anderen fallgerecht zusammengebracht werden. Dazu bedarf es eines Schemas, sei es eines Schemas reiner Sinnesbegriffe, empirischer Begriffe, reiner Verstandesbegriffe, oder gar eines der Idee. $4^{4}$ Schemata fungieren hier gleichsam als Regeln für die Anwendung von Regeln, genauer gesagt: sie sind Anwendungsbedingungen von Regeln. Insofern betrifft der Schematismus aus Kantischer Sicht ein schlechterdings notwendiges Vermittlungsprinzip im Verhältnis von Form und Inhalt, oder noch allgemeiner: von Prinzip und Prinzipiat. Das Prinzip bliebe sonst bloße Funktion, ohne einen Gegenstand vorstellen $\mathrm{zu}$ können; es käme nicht zum Gegenstand als einem geformten Inhalt; „ohne Schemate", wie es bei Kant heißt, blei-

\footnotetext{
${ }^{4}$ Zwar ist es richtig, daß Kant im Schematismuskapitel das Schema der Idee nicht erwähnt, aber offenbar schöpfen die ersten drei genannten Begriffsarten Kant zufolge den Schematismus nicht aus (versus Bunte 2016, 58 Anm. 219)). In der Regel wird nicht berücksichtigt, daß Kant auch ein Schema der Idee kennt (vgl. etwa auch Düsing (1995, 50 ff.), oder Höffe (2003, 154 f.)) Auch die Idee bedarf zur Ausführung eines „Schemas“ (KrV B 860, vgl. 693 f.).
} 
ben „Verstandeshandlungen“ ebenso wie die daraus entstehende "Vernunfteinheit" an sich selbst "unbestimmt" (KrV B 692 f.). Zwar hat der Schematismus einen allgemeinen Sinn, der über den spezifischen der vier genannten jeweils hinausgeht, gleichwohl muß gerade mit Blick auf die Weiterbildungen Kants im nachfolgenden deutschen Idealismus das Augenmerk zunächst auf die fundierende Ebene des Verhältnisses von Anschauung und Kategorie (reinem Verstandesbegriff) gerichtet werden.5 Sie betrifft nämlich auch die Grundstruktur der in Anschlag gebrachten Erkenntnisrelation (als einer von Anschauung und Begriff bzw. Form und Inhalt). Auf die transzendentalen Schemata als Ermöglichungsbedingungen der Erkenntnis kommt es auch Kant an.

Welches Problem hat das Lehrstück vom Schematismus der reinen Verstandesbegriffe zu bewältigen? Ein Konkreszenzproblem: im Schematismuskapitel muß nicht mehr, wie in der transzendentalen Deduktion, der rechtmäßige Gebrauch der Kategorien als Prinzipien gegenständlicher Bestimmtheit ausgewiesen werden, sondern einsichtig gemacht werden, wie Kategorien auf Gegenstände der Erkenntnis angewendet werden können (KrV B 177, 167). Immerhin schafft die kategoriale Synthesis des Verstan- des keinen Gegenstand, sondern dieser ist als ein zu bestimmender durch die Sinnlichkeit allererst gegeben. Deren universale Form ist die Zeit. Gegenstände erscheinen in der Zeit. Die Kategorie indes ist hinsichtlich des in der Zeit gegebenen Gegenstandes, auf den sie angewendet wird, Kant zufolge gänzlich „heterogen“ (KrV B 177), also nicht „gleichartig“ (KrV B 176). „Subsumtion" eines Gegenstandes unter einen Begriff setzt jedoch „Gleichartigkeit" voraus. Wie können Kategorien, rein logische, unzeitliche Begriffe auf in der Zeit gegebene Gegenstände angewendet werden? Dazu bedarf es Kant zufolge eines vermittelnden "Dritten“, d. i. einer "Vorstellung", die freilich rein sein muß und dabei "sinnlich" wie „intellektuell“ (apriorisch regelhaft): gleichartig mit der Kategorie und der Erscheinung eben (KrV B 177).

Im Hintergrund dieser Ungleichartigkeit, die durch das Schema überwunden werden soll, steht die von Kant ständig betonte Heterogenität von Anschauung und Begriff bzw. Sinnlichkeit und Verstand. Wie Kant an dieser Stelle sagt, sind reine Verstandesbegriffe im Vergleich mit empirischen, ja überhaupt sinnlichen Anschauungen "ganz ungleichartig" (KrV B 176), woraus sich dann auch die besagte Heterogenität von Kategorie und Erscheinung ergibt; diese setzt jene voraus.

\footnotetext{
${ }^{5}$ Vgl. zur systemphilosophischen Relevanz des Schematismus der Idee in der Transzendentalphilosophie Krijnen (2001, Kap. 7.3.2.1.2; 2008b, Kap. 6.1.2).
} 
Die „transzendentale Zeitbestimmung“ erfüllt nun die erforderliche Bedingung der Gleichartigkeit, ist sie doch einerseits wie die Kategorie „allgemein“ und gegründet auf einer „Regel a priori“ sowie anderseits mit der Erscheinung insofern gleichartig, als die „Zeit“ in jeder empirischen Vorstellung des Mannigfaltigen enthalten ist: die transzendentale Zeitbestimmung ist das Schema der Kategorie (KrV B 177 f.). Mit ihr liegt konkrete Gegenstandserkenntnis vor; sie ist in einem reine Synthesis wie unmittelbare Gegenstandsreferenz: Kontingentes ist zur kategorial fundierten Einheit gebracht, die die konkrete Gegenstandsbestimmung ist. Die Anwendung von Kategorien auf Erscheinungen als Gegenstände der Erkenntnis ist insoweit geklärt.

Die These der gänzlichen Heterogenität von Kategorie und Erscheinung, die zwecks konkreter Gegenstandserkenntnis eines vermittelnden Schemas bedarf, reicht auch für die im folgenden $\mathrm{zu}$ diskutierende Feststellung aus, daß ein solches Schema, jedenfalls als grundlegendes Definitionsstück der Erkenntnis, in der kantianisierenden Transzendentalphilosophie fehlt. ${ }^{6}$ Kants Schematismusproblem betrifft hier allenfalls ein nachgeordnetes, kein fundamentales Problem der Erkenntnislehre. Was sind die Gründe für diese Transformation?

Zunächst, um diesem Kalauer der
Kant-Forschung ebenfalls eine Reverenz zu erweisen, kann es sich nicht um eine Irrelevanz im Sinne der (sich an Curtius (1914) orientierenden) Kritik von Kemp Smith handeln. Sinnliche Anschauungen und Kategorien seien entweder nicht völlig heterogen, da sonst Subsumtion unmöglich wäre, oder sie seien es, dann erweise sich der Schematismus jedoch als überflüssig (Kemp Smith 1918, 318). Man hat Kemp Smiths Vorwurf nicht nur in die Nähe „Neukantianischer Interpretationen“ gerückt, sondern in Kemp Smiths Kritik zugleich einen ontologisierenden Aristotelischen hyletischen Sensualismus gewittert (Birrer 2017, 2 f. mit 17).Damit tun sich schon zwei wichtige, eng miteinander verbundene $\mathrm{Ge}$ sichtspunkte auf, die für eine Einschätzung des Südwestdeutschen Umgangs mit der Schematismusproblematik relevant sind.

Zum einen nämlich qualifiziert das Form-Inhalt-Verhältnis für die Südwestdeutschen die Geltungsstruktur der Erkenntnis. Insofern steht es jenseits allen Ontizismus eines sinnlichen Stoffes, der durch das Denken unsinnliche Formen erhalte, so daß ein Kosmos, ein geformtes Gebilde entstehe. Das Verhältnis betrifft, Kantisch gesprochen, zwei Arten von Vorstellungen zueinander: Anschauung als Vorstellung von Einzelnem und Begriff als Vorstellung von Allgemeinem.

\footnotetext{
6_ ebenso wie in Hegels spekulativem Idealismus.
} 
Es handelt sich somit bei Kant um ein vorstellungstheoretisch modelliertes Verhältnis von Vernunftvermögen, nicht um eine Ontologie von verschiedenen Seinsarten. Wie Kant sagt, ist die das „Mannigfaltige gegebener Vorstellungen“ (Anschauungen oder Begriffe) unter eine "Apperzeption" bringende „Handlung des Verstandes“ die „logische Funktion der Urteile"; also ist alles Mannigfaltige in Ansehung einer Urteilsfunktion ",bestimmt", durch die es nämlich zu einem „Bewußtsein“ gebracht wird; und da die "Kategorien“ nichts anderes sind als ebendiese Urteilsfunktionen (in ihrer Realfunktion), steht alles Mannigfaltige einer gegebenen Anschauung unter Kategorien (KrV B 143). Mit Hegel gesprochen, ist Sein eine Gedankenbestimmung, oder wie der Neukantianer Rickert sagt, Inhalt ist selbst eine Form, und Gegenstände sind immer Gegenstände der Erkenntnis. Das besagte Verhältnis bzw. Vermittlungsproblem betrifft also ein intragnoseologisches Verhältnis. Es bezieht sich zudem zunächst nicht auf das Verhältnis von Kategorie und Gegenstand, sondern auf das von Anschauung und Begriff, ist der Gegenstand als konstituierter doch immer schon das Ergebnis einer kategorialen Synthesis. Der Schematismus betrifft das Problem konkreter gegenständlicher Bestimmtheit (,Subsumtion von Gegenständen unter den Begriff'); Ka- tegorien werden den konkreten Gegenständen beigelegt, reine Verstandesbegriffe unter der Bedingung der Sinnlichkeit auf die Sinnlichkeit angewendet. So wird plausibel, warum wir Grundsätzliches (synthetische Urteile a priori) über die Natur als Gegenstand der Erkenntnis aussagen können. Aus den reinen Verstandesbegriffen fließen synthetische Urteile a priori, die sodann als Grundsätze jedweder Gegenstandserkenntnis fungieren. Es sind dies Prinzipien, die die Gegenstandsbestimmung konstitutiverweise in ihrer Konkretion begründen. Die Kategorie ist also nicht nur überhaupt auf Sinnlichkeit bezogen, sondern konkretisierend auf Sinnlichkeit bezogen, ihre Bestimmtheit vereinzelt sich.7 Kategoriale Bestimmtheit wird zur Vereinzelung der Bestimmtheit führende generelle Bestimmtheit. Diese Vereinzelung gilt es im Schematismuskapitel zu begreifen. In ihr wird Heterogenes, die Intellektualität der Kategorie und die anschauliche Mannigfaltigkeit des (inneren) Sinnes, repraesentatio generalis und repraesentatio singularis, in ein Vermittlungsverhältnis gebracht. Das Schema ist das ,Dritte', die beide begründungsfunktional vermittelnde begrifflich-apriorische Vorstellung. Die apperzeptionstheoretisch fundierte Einheit der Synthesis wird mit der einzelnen Anschauung vermittelt, so daß „Einheit in der Bestim-

\footnotetext{
${ }^{7}$ Flach $(2001 ; 2002,160$ ff.) hat dies in seiner Interpretation der Kantischen Schematismuslehre betont.
} 
mung der Sinnlichkeit“ entsteht (KrV B 179). So kommt es bei Kant zur Bestimmung der Empirie als gesetzmäßig. Die Schematismuslehre entwickelt damit die der reinen Verstandesgesetzlichkeit untergeordnete Gesetzmäßigkeit der Empirie: konkrete Gegenstandsbestimmung (Naturerkenntnis) ist für Kant gesetzmäßige Bestimmung.

Zum anderen wird das Erkenntnisverhältnis bei den Südwestdeutschen nicht mehr, wie bei Kant, vorstellungstheoretisch expliziert, sondern geltungsstruktural: Erkenntnis ist hinsichtlich der Struktur ihrer Geltung thematisch. Diese Struktur ist eine Struktur nicht von Vermögen, sondern von Geltungsfunktionen. Kants Erkenntnis, daß Erkenntnis sich in Urteilen vollzieht und Begriffe auf Funktionen zu Urteilen beruhen, führt dazu, die Erkenntnisqualifikation als Inbegriff von Prinzipien, Geltungsfunktionen gegenständlicher Bestimmtheit zu konzeptualisieren. Kant gibt dieser rein bestimmungslogischen, am Urteil orientierten und in konstitutiver und regulativer Hinsicht abgehandelten Thematik der Gegenstandsbestimmung im Zuge seiner vorstellungstheoretischen Modellierung jedoch zugleich eine apperzeptionstheoretische Pointierung, indem er die Bestimmungskom- petenz des ,ich denke' in die Erkenntnisgrundlegung integriert. 8 Es ist systematisch gesehen nicht zuletzt Kants eigene Unterscheidung einer subjektiven von einer objektiven Deduktion, die in der nachfolgenden Transzendentalphilosophie zum ,Primat' einer ,geltungsnoematischen' oder ,objektivlogischen', die Prinzipien des Erkenntnisgehalts betreffenden Erkenntnisfundierung geführt hat (Krijnen 2008a; 2014a). Infolgedessen ergibt sich die Thematik des sich den Gegenstand der Erkenntnis aneignenden Subjekts bestenfalls als Anschlußthematik; die Funktion des ,ich denke' als höchster Apperzeptionseinheit wird geltungsnoematisch expliziert..$^{9}$

\section{Das südwestdeutsche Bedürfnis nach der Vereinheitlichung des Kan- tischen Dualismus}

\begin{abstract}
Daß die Neukantianer Kant primär aus einem systematischen Blickwinkel thematisieren, ist oben schon angedeutet worden. Obwohl Neukantianer wie Cohen $(1889 ; 1910 ; 1918)$, Bauch (1923a) oder Cassirer (1994a, 585-762; 1994b) zugleich Kant-Exegeten sind,
\end{abstract}

\footnotetext{
${ }^{8}$ Nicht umsonst legt Flach, der womöglich konsequenteste Verfechter einer rein geltungsfunktionalen Erkenntnisgrundlegung (vgl. Flach 1994), in seiner Kant-Deutung auf diesen Punkt den Finger (Flach 2002, 112 ff.).

${ }^{9}$ - und bei Hegel ist die Logik ohnehin Ideenlehre im abstrakten Element des Denkens; Realverhältnisse bzw. ein erkennendes Subjekt spielen hier ebenfalls noch keine Rolle; die Funktion des ,ich denke' als transzendentaler Apperzeptionseinheit wird vom spekulativ verstandenen Begriff übernommen; ein reales Ich indes ist Thema erst in der Philosophie des subjektiven Geistes.
} 
bringt der spiritus rector der südwestdeutschen Neukantianer Windelband (1915, IV) den neukantianischen Umgang mit Kant schon früh einprägsam auf den Punkt: „Kant verstehen, heißt über ihn hinausgehen“, d. h., ihn systematisch weiterbilden.10 Sachlich gesehen kreisen die zentralen Gedanken des Neukantianismus in seiner Marburger und südwestdeutschen Ausprägung um das Problem der Geltung. Kants Beitrag zur Philosophie schätzen die Neukantianer sowohl hinsichtlich seiner Einsicht in das Problem der Geltung als auch in die Methode, mit der das Geltungsproblem bewältigt werden kann. Dieser Beitrag Kants soll nicht bloß reaktiviert, sondern zugleich reaktualisiert werden.

Für die Neukantianer handelt es sich beim Transzendentalen Kants der Sache nach um einen Inbegriff von Geltungsgründen, der nicht durch den Rückgang auf ein Seiendes außerhalb der Erkenntnisrelation begriffen wird, sondern durch einen Rückgang auf das Denken als Grund aller Geltung. Die objektive Gültigkeit konkreter Sinnleistungen des Subjekts findet ihren Grund in einem Inbegriff von Geltungsprinzipien (,Bedingungen der Möglichkeit'); die objektive Gültigkeit dieser Geltungsprinzipien wird dadurch legitimiert, daß sie sich geltungsfunktional als Bedingungen der Erkenntnis (letzt-) begründen lassen. Wie Kant sind die Marburger und Südwestdeutschen also darum bemüht, die Bestimmtheit der Erkenntnis durch die Bestimmung der Geltungsbestimmtheit des Wissens zu ermitteln. Indem sie Kants Transzendentalphilosophie als Geltungstheorie deuten, werden ,metaphysische' Reste und ,psychologische' Depravationen Kants getilgt bzw. der geltungstheoretischen Kernaussage subordiniert. 11

Mit Blick auf die erste Dimension des Schematismusproblems - den fundierenden Hintergrunddualismus von Anschauung und Begriff bzw. Sinnlichkeit und Verstand -, ist Kants transzendentale Ästhetik nicht nur in der Frühphase des deutschen Idealismus unter Beschuß geraten, sondern eben

\footnotetext{
${ }^{10}$ Vgl. auch: Cohen (1902, VII): „Von vornherein war es mir um die Weiterbildung von Kant's System zu thun“. Rickert (1924/25, 163-66): Neukantianer dürfen sich nur diejenigen nennen, die etwas Neues gebracht haben und also „dadurch, daß sie auf Kant zurückgingen, die wissenschaftliche Philosophie zugleich vorwärts" führten; entsprechend kommt es Rickert (1899, Vorbemerkung) nicht darauf an, was ,kantisch', sondern darauf, was ,richtig' ist. Natorp (1974, 243): Es wird nicht „etwa diese oder jene Aufstellung Kants starr festgehalten“, sondern es geht darum, daß „die Bearbeitung der Aufgabe, die er der Erkenntniskritik [...] mit Klarheit gestellt hat, mit gesammelter Kraft aufgenommen, was er und die andern Großen vor und nach ihm zur Lösung dieser Arbeit beigetragen haben, gewissenhaft genutzt, geklärt, vertieft und weitergeführt wird“. Kants Philosophie bilde keinen „,vom Himmel gefallenen Gesetzeskodex“ (Natorp 1912, 194), sondern die Marburger Neukantianer seien überzeugt von den „notwendigen Korrekturen an der Lehre Kants" $(1912,196)$. - Freilich sollte man sich in bezug auf die Kant-Interpretation des Neukantianismus vor der Suggestion allzugroßer Homogenität hüten (schon Cohens Kant-Deutung wurde sowohl innerhalb der Marburger Schule als auch seitens der Südwestdeut-schen kritisiert). Das Verhältnis der Neukantianer zu Kant kommt in vielen Arbeiten zum Neukantianismus zur Sprache. Vgl. zum Thema etwa Heinz und Krijnen (2007).

${ }^{11}$ Vgl. zur Vielschichtigkeit des transzendentalen Gedankens bei Kant etwa Zocher $(1959,20-48)$ der selbst dem südwestdeutschen Neukantianismus entstammt. Neuerdings hat der Kant- und Neukantianismuskenner sowie Philosophiesystematiker Flach von einer „doppelten ontologisch-vermögenstheoretischen Hypothek“ Kants gesprochen, die die nachkantische Geltungs- und Prinzipienlehre vermeiden müsse (Flach 2015, hier 24).
} 
auch im Neukantianismus. Während Hegel (1971, Bd. 20, 339) zufolge Kant die beiden Stämme der Erkenntnis nicht systematisch, also "aus dem Begriff“ entwickelt, sondern „empirisch“, vor allem aus der Psychologie aufnimmt, werfen die Neukantianer Kant mutatis mutandis dasselbe vor. Und zweifelsohne ist Kants Philosophie problemgeschichtlich vor dem Hintergrund des schulphilosophischen Rationalismus und des britischen Empirismus entstanden; in diesem Kontext wirft Kant seine Frage nach der ,Beziehung der Erkenntnis auf ihren Gegenstand' auf und beantwortet sie durch die Kritik der reinen Vernunft. In einer anderen problemgeschichtlichen Lage indes befinden sich die Neukantianer: ihre philosophische Zeit ist gekennzeichnet durch den Niedergang des nachkantischen Idealismus und den Aufstieg des deutschen Empirismus; durch einen Rückgang auf Kant wollen die Neukantianer sozusagen beide , aufheben'. Daß ihre Auseinandersetzung mit und Verarbeitung von Kant zu einem anderen systematischen Entwurf führen mußte, ist daher vorprogrammiert.

Die erste Komponente, die für die Südwestdeutschen an Kants transzendentaler Ästhetik unbefriedigend ist, betrifft eben jenen basalen Hintergrundgrunddualismus. Sie betrifft also das konstitutionstheoretische Problem der transzendentalen Ästhetik selbst, und damit das Problem von Sinn, Funktion und Begründung beider ,Stämme' (KrV B 29) der Erkenntnis. Im Kontext meiner Problemstellung reicht es aus, hier nur auf die (auch) im südwestdeutschen Neukantianismus wirksame Fortbildungstendenz des anfänglichen Kantischen Stämmedualismus hinzuweisen, $\sqrt{12}$ die sachlich in Rickerts ,Modell eines theoretisch gedachten Gegenstandes überhaupt', d. i. in seinem sog. ,Gegenstandsmodell', kulminiert (1921, 50 ff.; 1924, 10 ff.). Hier stellt sich heraus, daß Form und Inhalt zum Begriff dessen gehören, was gedacht wird, wenn etwas überhaupt theoretisch gedacht wird. Mit diesem Inhalt ist noch gar kein besonderer (dieser, jener) Inhalt gemeint, sondern Inhalt selbst erweist sich als Bestandteil des Gegenstandmodells, gehört zu den formalen Faktoren des theoretischen Gegenstandes überhaupt. Auf dieser fundamentalsten Ebene der Selbstbeziehung objektiven Denkens auf Inhalt spielen besondere Formen wie Raum und Zeit keine Rolle: ,Inhalt überhaupt' ist vielmehr, wie Rickert sagt, der ,logische Ort für das Alogische', garantiert die Inhaltsbezogenheit des Denkens und ermöglicht dadurch eine Mannigfaltigkeit von Inhalten.

Damit ist zwar ein anderes, einheitlicheres Konstitutionsmodell der Erfahrung ursprungstheoretisch oder

${ }^{12}$ Vgl. zur Thematik ausführlich Krijnen (2007). 
primär-konstitutiv auf den Weg gebracht, dieser erkenntnistheoretische Sachverhalt bildet jedoch zugleich die Grundlage für die logisch nachgeordnete Aufgabe einer wissenschaftstheoretischen Grundlegung einzelwissenschaftlicher Erkenntnis. Diese betrifft die zweite Komponente der Kritik. Sie besagt: Kants transzendentale Ästhetik restringiere mit den Formen Raum und Zeit das der Erkenntnis unmittelbar Gegebene auf sinnlich Gegebenes. Für Rickerts Erkenntnis- und Wissenschaftstheorie wie seine Ontologie ist diese zweite Komponente nicht weniger wichtig als die erste. Beide Komponenten sind essentielle Bestandteile südwestdeutscher Rezeption von Kants transzendentaler Ästhetik der Kritik der reinen Vernunft. Es handelt sich bei der zweiten Komponente nicht um die Erkenntnisfunktion der Anschauung aus $\S 1$, sondern um die in den $\S \S 2$ ff. eruierten Formen des Angeschauten. ${ }^{13}$

Beide Komponenten sind in je verschiedener Weise für das Schematismusproblem relevant. Die erste Komponente betrifft Kants Stämmedualismus versus das für die Südwestdeutschen maßgebende und von Rickert in bestimmtester Weise auf den Weg gebrachte Lehrstück der Heterologie. Kants Stämmedualismus und Rickerts Heterologie betreffen jeweils die grundlegende Struktur der Erkenntnis qua geltungsfunktionales Modell des Wissens.
Bei beiden handelt es sich um ein zweigliedriges Ganzes. Kant denkt sich dieses Ganze als bestehend aus „zwei Stämmen der menschlichen Erkenntnis“ („Sinnlichkeit und Verstand“), wie er immer wieder betont, auch schon unmittelbar vor Beginn der, transzendentalen Ästhetik' (KrV B 29). Er entwickelt die Bestimmung dieser zwei Stämme weiter in $\S 1$ der, transzendentalen Ästhetik' und kommt am Anfang der ,transzendentalen Logik' auf sie zurück, wo er von „zwei Grundquellen des Gemüts“ („Rezeptivität“ und "Spontaneität“) bzw. „Anschauung“ und „Begriff" als den „Elementen aller unsrer Erkenntnis" spricht $(\mathrm{KrV}$ B 74). Die Erkenntnis qualifiziert sich durch die Momente der ,Anschauung' und des ,Begriffs' bzw. der ,Sinnlichkeit' und des ,Verstandes' bzw. der ,Rezeptivität' und der ,Spontaneität'. Diese „zwei Stämme“ (KrV B 29) der Erkenntnis machen ihre Fundamentalbestimmtheit aus; sie sind die grundlegenden Prinzipien der Erkenntnis. In und mit diesen Prinzipien konstituiert sich die Erkenntnis. Die Erkenntnis ist ein geltungsfunktionales Bedingungsgefüge der Prinzipien der Anschauung und des Begriffs, von aisthetischen und logischen Bedingungen.

Damit oszilliert die Erkenntnis ihrer Geltungsstruktur nach zwischen Unbestimmtheit und Bestimmtheit: Die aisthetischen und logischen Bedin-

${ }^{13}$ Vgl. dazu eingehender Krijnen (2013). 
gungen der Erkenntnis konstituieren sowohl die Gegebenheit eines Gegenstandes als auch das Denken eines Gegenstandes (KrV B 29 f., 74 f.). Durch die Sinnlichkeit wird der Gegenstand gegeben, durch den Verstand gedacht. Entsprechend gibt es Prinzipien (,Formen'), die die Erkenntnisfunktion der Anschauung sicherstellen: die Anschauungsformen Raum und Zeit; und Prinzipien, die die Erkenntnisfunktion des Denkens sicherstellen: die reinen Verstandesbegriffe (Kategorien). Diese beiden elementaren Erkenntnisfunktionen definieren die Erkenntnis grundlegend in zwei Konstitutionsrücksichten. Beide wirken in der Konstitution der Erkenntnis zusammen: „Gedanken ohne Inhalt sind leer, Anschauungen ohne Begriffe sind blind. [...] Beide Vermögen, oder Fähigkeiten, können auch ihre Funktionen nicht vertauschen. [...] Nur daraus, daß sie sich vereinen, kann Erkenntnis entspringen." (KrV B 75).

Die ,transzendentale Ästhetik' thematisiert die Erkenntnis qua Vorstellung von einem unbestimmten Gegenstand als Einzelvorstellung (repraesentatio singularis): Anschauung. Die Anschauung repräsentiert den Gegenstand unvermittelt. Die Erkenntnisbedeutung der Anschauung bzw. der Sinnlichkeit oder Rezeptivität besteht darin, Material für die Erkenntnis bereitzustellen; sie etabliert den unbestimmten, zu bestimmenden Gegenstand, also ein Substrat möglicher Bes- timmungen, angefangen mit der „Empfindung" (sensatio, Sinneseindruck) als genetisch gesehen unterster Ebene von „Vorstellung mit Bewußtsein" (KrV B 376), die qua „Wirkung eines Gegenstandes auf die Vorstellungsfähigkeit, sofern wir von demselben affiziert werden" (KrV B 34), für sich genommen noch völlig geltungsindifferent, bloß ,subjektive Perzeption' (KrV B 376), Materie der Erkenntnis (vgl. $\mathrm{KrV} \mathrm{B}$ 34 mit 74) ist. Die Anschauung, von der bei Kant die Rede ist, ist freilich nicht mehr geltungsindifferente Empfindung von Eindrücken, sondern bereits apriorische Geformtheit des gelieferten Materials. Die empirische Anschauung kann nur deshalb Erkenntnisrelevanz besitzen, weil die ,reinen' Anschauungen den empirischen einen Gegenstandsbezug ermöglichen. In dieser Funktion des unmittelbaren Gegenstandsbezugs ist die Anschauung der vermittelten Gegenstandsrepräsentation durch den Begriff sowohl entgegengesetzt als auch zugeordnet. $\mathrm{Zu}$ sammen mit ihrem geltungsfunktionalen Gegenstück ,Denken' macht die Anschauung die Bestimmtheit des Erkenntnisgehalts aus.

Zwar handelt es sich bei den Anschauungsformen Raum und Zeit um etwas mit Anschauungscharakter und nicht mit Begriffscharakter, so daß die Anschauungsformen nicht mit den Kategorien identisch sind. Gleichwohl sind die Anschauungsformen geltungsfunktional darauf angelegt, die Gel- 
tungsrelevanz des Materials für die Erkenntnis zu sichern. Im geltungsfunktionalen Gefüge der Erkenntnis übernehmen sie eine konstitutive Funktion. Das konstitutionstheoretische Verhältnis von Anschauung und Begriff ist folglich dergestalt entwickelt, daß der Bezug zur Sinnlichkeit prinzipielles Ermöglichungsmoment der Erkenntnis ist. Die reine Anschauung ist bei Kant Form - und damit Prinzip von Verhältnissen, apriorischer Faktor möglicher Gegenstandsbestimmung ( $\mathrm{KrV}$ B 34 f.), ${ }^{14}$ darin unterscheiden sich Anschauung und Begriff nicht. Nur gemeinsam ergeben reiner Begriff und reine Anschauung die apriorische Ermöglichung gegenständlicher Bestimmung.

Die zwei ,Stämme' der Erkenntnis sind qua Erkenntnisquellen in ihrer Funktionalität für die Erkenntnis, in ihrer Geltungsrelevanz gefaßt. Daher sind sie zugleich dem Einen Prinzip der Erkenntnis subordiniert und rein transzendentalphilosophisch gedeutet. Die kognitive Relation, die Erkenntnisbeziehung zeichnet sich ihrem Sinn nach nicht durch eine Hypostasierung von zwei irreduziblen Elementen aus, sondern durch das geltungsfunktionale Zusammengehören von zwei Momenten des Einen, das die Erkenntnis ist. Es sind im wesentlichen die beiden Momente der Unbestimmtheit (unbegriffenen Mannigfaltigkeit) und Bestimmtheit der Erkenntnis. Dies jedenfalls ist der sachliche Kern von Kants Aussage. An ihm halten auch die Südwestdeutschen fest. Mit Kants Durchführung dieses Gedankens sind sie jedoch unzufrieden.

\section{Die Südwestdeutschen über Sche- matismus und ursprüngliche Einheit}

(1) Der südwestdeutsche Neukantianer Bauch, der vielleicht beste der neukantianischen Kant-Interpreten, hat sich in seiner umfassenden Kant-Deutung (1923a) sowie in seinem systematischen Hauptwerk zur theoretischen Philosophie (1923b) auch zum Schematismusproblem geäußert.15 Schon die Kant-Deutung kehrt in puncto Fundamentalstruktur der Erkenntnis Entscheidendes hervor, und zwar zunächst bereits im Kontext von Ausführungen zur transzendentalen Ästhetik:

Zum einen bemüht sich Bauch, das erkenntnisfunktionale Anliegen der transzendentalen Ästhetik herauszuarbeiten (1923a, 152 ff.): die thematische Geltungsfunktion erweist sich

\footnotetext{
${ }^{14}$ Die Form der Anschauung ist das, was es ermöglicht, die ,Empfindungen“, ,Materie‘ oder das „Mannigfaltiᄀge der Erscheinung“ [als das Mannigfaltige der Anschauung in seiner Unbe $\neg$ stimmtheit, ck] zu ,ordnen' (KrV B 34).

${ }^{15}$ Vgl. zu Bauchs Modifikationen diesbezüglich neuerdings auch Pringe (2015). Pringe streicht dabei die Bedeutung des Infinitesimalprinzips für Bauchs Lösung des Schematismusproblems heraus. Allerdings endet Bauchs Argument gegen Kants Ansatz des Schematismus auf S. 270 (Bauch 1923b, 270). Das Infinitesimalprinzip spielt erst eine Rolle bei der Interpretation der ,Grundsätze bzw. der Ausgestaltung der kategorialen Bestimmtheit der Empfindung als Quantität (Bauch 1923b, 272 f.).
} 
als die der Etablierung eines Bestimmungssubstrats. Zum anderen argumentiert Bauch gegen einen radikalen Stämmedualismus der Erkenntnis. Beide ,Stämme' beträfen geltungsfunktional gesehen unterschiedliche objektive „Geltungsbedingungen“ und „Geltungsanteile“: auch die Prinzipien der Sinnlichkeit thematisiere Kant unter dem „objektiven Gültigkeitsgesichtspunkt" (Bauch 1923a, 153). Aufgrund ihrer eigenen Aufgabe könne die Sinnlichkeit für eine „Wissenschaft von allen Prinzipien der Sinnlichkeit a priori“ (KrV B 35) trotz aller ursprünglichen Dualität von Denken und Anschauung nicht völlig alogisch sein; vielmehr deutet sich hier Bauch (1923a, 154) zufolge der Gedanke eines „Logischen im Alogischen“ an, komme der Anschauung Erkenntnisbedeutung doch nur durch den „Begriff der Erkenntnis“ zu: der Begriff der Erkenntnis ermögliche die Aufgabe der transzendentalen Ästhetik als einer transzendentalen. Entsprechend läßt Bauch (1923a, 155 f.) die psychologische Färbung des Kantischen Begriffsinstrumentariums gegenüber der logischen oder geltungsfunktionalen Stelle des Gedankens im Begriff der Erkenntnis zurücktreten.

Durch die Empfindung werde der Gegenstand zwar nicht "bestimmt", das Material der Empfindung sei jedoch erkenntnisrelevant, das Aposteriori also dem Logischen qua Sphäre der Form zugehörig. Daß uns Material der Empfindung gegeben ist, ist Möglich- keitsbedingung und damit formale Bestimmtheit der Erkenntnis - das Aposteriori selbst Apriori: Materie und Form gehören intrinsisch zusammen (Bauch 1923a, 157 f.). Während die Materie uns ihrem materiellen Gehalt nach „gegeben" sei, mache die „Form" es möglich, daß das Mannigfaltige der Erscheinung, wie Kant sagt, geordnet werden kann (KrV B 34). Für Bauch liegt in diesem ,Machen' zugleich der Hinweis auf den Zusammenhang von Anschauung und Denken und damit auch auf den von transzendentaler Ästhetik und transzendentaler Logik (1923a, 159). Das Apriori steht bei Bauch für die "logisch-gesetzliche Bedeutung" als Möglichkeitsbedingung der Erkenntnis (1923a, 159 f.) und damit für den Zusammenhang zwischen Rezeptivität und Spontaneität, von der Materie der Empfindung über die Formen der Anschauung bis hin zur Form des Denkens. Nur weil die Form der Spontaneität als der Sphäre des Logischen angehörend noch die Materie der Empfindung beherrsche, könne diese überhaupt einen logischen Ort im Begriff der Erkenntnis erhalten (Bauch 1923a, 159 f., Anm. 4). Zwar arbeitet Bauch den logischen Zusammenhang von Form und Materie in seinem systematischen Gehalt klar heraus, damit geht jedoch ein ganzes Bündel von Vorwürfen an die Adresse Kants einher, auch wenn Bauch diese Vorwürfe, gerade was die fundamentalen Aspekte betrifft, immer wieder durch eine Art ,Buchstabe - 
Geist'-Strategie relativiert. Wie von der idealistischen Auseinandersetzung mit Kant bis zur heutigen Kant-Forschung moniert, sieht auch Bauch (1923a, 147 f.) Kants Lehre von den zwei Stämmen trotz ihres objektiv-logischen Sinns mit einem ontischen Einschlag befrachtet. ${ }^{16}$ Die Dualität bleibe jedenfalls für die Kritik der reinen Vernunft maßgebend, auch wenn Kant auf die Verbindung der beiden Geltungsfunktionen in der konkreten Erkenntnis hinarbeite, die Möglichkeit der Erkenntnis selbst die Einheit fordere (Bauch 1923a, 148, vgl. 153, 156). Und obwohl Bauch konzediert, Kants Methode der ,Isolierung' von Form und Materie der Sinnlichkeit sowie von Sinnlichkeit und Verstand $(\mathrm{KrV} \mathrm{B} 34,36)$ solle keine sachliche Trennung von Zusammengehörigem sein, sondern eine methodische der Betrachtungsweise (Bauch 1923a, 158 f.) ${ }^{17}$ hält er hinsichtlich der Kritik der reinen Vernunft den Isolierungsvorwurf für triftig (1923b, 2 f.). Die
Isolation führe $\mathrm{zu}$ einer Abstraktion, da Kant in der Kritik der reinen Vernunft (gerade auch in der ,transzendentalen Ästhetik') an der Selbständigkeit bzw. Unabhängigkeit von vorliegendem empirischem Material und logischen Formen festhalte.18 Dagegen könne es ein dem logischen Prinzip gegenüber schlechthin unabhängiges und gleichursprüngliches Prinzip (,gegebenes Material') nicht geben (1923a, 200 f. mit 204). Wie Bauch darlegt, haben die jeweiligen Glieder ihre Bestimmtheit nur in bezug aufeinander. Ein „schroffer Dualismus von ,Form' und ,Inhalt'“ entstelle daher die Sachlage (1923a, 203). Entsprechend klagt Bauch (1923a, 203) die „objektive Vernunft" als Grundlage ein. Kants Festhalten am ,Ding an sich' ist ihm logisch gesehen nichts als der vergebliche Versuch, die Unabhängigkeit des Gegenstandes vom Subjekt zu sichern: auch diesbezüglich will Bauch den Geltungsanspruch des empirischen Mate-

\footnotetext{
${ }^{16}$ Auch Zocher diagnostiziert trotz der transzendentalen oder ,semantischen' Ausrichtung Kants eine „Ontik“ (einen ontischen Idealismus), die in den Varianten des „metaphysi-sschen“, „psychologischen“ und „vagen“ Idealismus auftrete (1959, $41 \mathrm{ff} . \mathrm{mit} 47$; vgl. auch Zocher 1954, 180 f., 190 ff.). Flach $(2015,21)$ hat bekanntlich von einer doppelten ontologisch-vermögenstheoretischen Hypothek gesprochen, in der u. a. das Begriffspaar Anschauung und Begriff (Kategorie) zu einem Begriff mit „geltungsdeduktiv destruktivem ontologischem Verweisungsgehalt" degeneriere, schon deshalb, weil das Vermögen auch als Habitus und damit als „ontische Beschaffenheit“ des Menschen aufgefaßt werde.

${ }^{17}$ Heidemann (2002, 78 ff.), bewertet Kants Methode der Isolation zwar positiv, muß jedoch eingestehen (2002, 98), daß sie sich das Problem einer eigenen Theorie der Vermittlung von Anschauung und Begriff, nämlich des Schematismus, in der konkreten Gegenstandserkenntnis einhandelt, so daß die ursprüngliche Heterogenität und Irreduzibilität von Anschauung und Begriff nicht strikt sein kann. Heidemanns Vorschlag der Begründung des Kantischen Stämmedualismus vermag es allerdings nicht, die Vorausgesetztheit der Differenz von Anschauung und Begriff zu tilgen. Vielmehr handelt Heidemann sich das Pro $\neg$ blem ein, daß der Anfang, um mit einer Hegelschen Reinhold-Kritik zu sprechen, bloß hypothetisch ist (vgl. Hegel 1971, Bd. 9, § 10 A; Bd. 5, 68 ff.). Indes gelingt die Begründung der Stämme nur aus dem geltungsfunktional verstandenen Begriff der Erkenntnis selbst, als der ,höheren' oder ,ursprünglichen' Einheit beider, diesie ist. Wenn Heidemann schließlich auf Brandoms Theorie diskursiver Praxis sowie der damit verbundenen Deutung von Begriffen als Funktionen und Normen (Brandom 1994) hinweist und damit die Möglichkeit einer Einheitlichkeit von Anschauung und Begriff suggeriert, redet er damit implizit dem Anliegen des südwestdeutschen Neukantianismus das Wort.

${ }^{18}$ Bauch hat sich umso mehr um ein streng funktionales Verständnis des Begriffs bemüht und verdient gemacht (vgl. Bauch 1914; 1923b; 1926).
} 
rials durch die Notwendigkeit des Begriffs gewährleistet wissen (1923a, 163 f. Anm. 1 mit 164 f.; 1923b, 204). ${ }^{19}$

Kurzum: Der anfängliche Stämmedualismus ist das Mißliche. An dessen Stelle hat ein ursprünglicheres Verhältnis zu treten. Bauch bringt der transzendentalen Revolution der Denkungsart gemäß die Vernunft als Grundlage in Anschlag, und zwar als ,objektive Vernunft'.20 Die ,objektive Vernunft' bildet die Grundlage: nur in ihrem Zusammenhang kann die Empfindung Erkenntnisrelevanz, logische Bedeutung gewinnen (Bauch 1923b, 204 ff.; vgl. 225 ff., 233 ff.). Gemäß Bauchs durchgängig funktionaler Bestimmtheitstheorie ist das ,Material', die ,Empfindung' u. dgl. selbst immer schon kategorial bestimmt, um Material und Empfindung sein zu können (1923b, 201 ff., 259 u. ö.): ohne kategoriale Gesetzlichkeit keine anschaulichen Gegenstände. Ein Sachverhalt wie kategorial unbetroffenes Material ist Bauch also eine "gänzlich leere Abstraktion", als Material undenkbar (1923b, 259).21 Kants berühmte Sentenz, „Gedanken ohne Inhalt sind leer, Anschauungen ohne Begriffe sind blind" ( $\mathrm{KrV}$ B 75), kann also kei- neswegs heißen, daß es leere Gedanken oder blinde Anschauungen überhaupt soll geben können, sind beide doch nichts als bloße Abstraktionen, vollzogen in der Reflexion; wie Bauch sagt, hat die Leerheit von Gedanken bzw. Blindheit von Anschauungen nur Sinn als deren Reinheit, also als (je differente) objektive Geltungsgesetzlichkeiten (1923b, 267; vgl. 1982, 266). Darin, objektive Geltungsgesetzlichkeit zu sein, kommen Anschauung und Begriff „prinzipiell“ überein (Bauch 1923b, 268).

Die Anschauung bzw. die Anschauungsgesetzlichkeit steht bei Bauch für die Geltungsfunktion des Einbezugs der „Empfindungsinhaltlichkeit“, des „mannigfaltigen Materials" in den kategorialen Geltungszusammenhang zur Bestimmtheit der Anschauung nach dem "Gesetz des Begriffs" (1923b, 259 mit 267 f.; vgl. 1926, 206 f.). Von den begrifflichen Gesetzlichkeiten des Kategorienzusammenhangs „prinzipiell“ verschieden oder getrennt kann die Anschauungsgesetzlichkeit, wie gesagt, nicht sein, handelt es sich doch stets um objektive Geltungsgesetzlichkeit; als Gesetzlichkeit ist die Anschauungsgesetzlichkeit immer schon kategoriale Gesetzlichkeit (1923b, 267 ff.).

\footnotetext{
${ }^{19}$ Vgl. zur Kant-Kritik auch: Bauch 1914. Auch hier bemängelt er Kants Fassung des Verhältnisses von empirischem Inhalt und kategorialer Form in der Kritik der reinen Vernunft $(1914,310)$ und des Dualismus von Anschauung und Kategorie sowie einen „verfehlten Ding-an-sich-Dogmatismus“ (1914, 332). In der ersten Kritik bleibe die „ursprünglich zwiespältige Position von Allgemeinheit und Besonderheit, von Form und Materie der Erkenntnis überhaupt, wie innerhalb der Form von Begriff und Anschauung“ nolens volens erhalten $(1914,336)$.

${ }^{20}$ Vgl. für Bauchs Systematik vor allem Wahrheit, Wert und Wirklichkeit (1923b). Vgl. zudem Bauchs Die Idee (1926) für das Fortschreiten zur Ideenlehre sowie Bauchs Theoretische Philosophie (1982). Vgl. dazu Krijnen (2008b, Kap. 5) sowie González Porta (1990).

${ }^{21}$ Vgl. auch Bauch (1926, 203 f.), wo er das isolierte Denken des Inhalts der Empfin $\neg$ dung als abstrakte Handhabe kritisiert.
} 
Daher wird bei Bauch aus der Unterscheidung von Geltungsgesetzlichkeiten keine „Isolation“, die dann eines Mittlers bedürfte. Die Vereinigung läßt sich vielmehr schon in der transzendentallogischen Bedingtheit des konkreten Gegenstandes der Anschauung aufweisen (1923b, 270).22 Das Material der Erkenntnis selbst ist in die Formen der Geltungsbeziehungen objektiven Denkens einbezogen.

In ebendiesem Kontext äußert sich Bauch auch explizit zum Schematismusproblem bei Kant. Kant gelange nicht $\mathrm{zu}$ einem adäquaten Verständnis des Verhältnisses von Anschauung und Begriff, was "am bezeichnendsten“ im Schematismus zutage trete (Bauch 1923b, 270). Denn zwar seien Raum und Zeit keine Kategorien bzw. Begriffe, aber die Unterscheidung von reiner Anschauung und reinem Begriff werde Kant zu einer „Isolation“, die eines „Mittlers" bedürfe, um das Getrennte wieder $\mathrm{zu}$ vereinen, obwohl, wie von Kant freilich erkannt, ohne eine solche Vereinigung weder ein Gegenstand der Anschauung noch die Anschauung eines Gegenstandes möglich sei (1923b, 270). Reine Anschauung und Kategorie bzw. Begriff seien nicht voneinander geschieden, sondern gehörten in der "transzendentallogischen Bedingtheit des konkreten Gegenstandes" zusammen, bedürf- ten also keiner „künstlichen“ Vereinigung (1923b, 270). Und ähnlich heißt es in Bauchs Kant-Buch, daß Kant anfänglich Anschauung und Begriff „gänzlich ,isoliert“" habe, während das Isolierte zwar in der „Abstraktion" und "Reflektion“ isoliert, in der Erkenntnis jedoch nicht getrennt werden könne (1923a, 233); diese Vorgehensweise räche sich in Kants Lehre von den Grundsätzen, weil die ursprüngliche Isolation nicht bloß als Reflexionsprodukt, sondern als sachliche Konstellation in Anspruch genommen werde; das Problem der "Anwendung" stelle sich entsprechend so dar, als müßten Begriff und Anschauung, die durch die Begriffe des Transzendentalen und der Synthesis schon vereint sind, erst noch durch ein Drittes, den Schematismus, zusammengebracht werden (1923a, 234). Kurzum: Bauch zufolge rächt sich Kants Isolations- und Abstraktionsverfahren dergestalt, daß ein „künstlicher" Vermittler vonnöten ist (1923a, 234; vgl. 239). Von dieser „Einstellung" her thematisiert Bauch (1923a, 234 ff.) das Schematismuskapitel. Den Gedanken einer völligen Ungleichartigkeit von Begriff und Anschauung lehnt er also ab (1923a, 235). Gerade daraus aber ergab sich bei Kant die Frage nach der Anwendung der Kategorie auf Erscheinungen. Sie muß Bauch $(1923 a, 236)$ als „sinn-

\footnotetext{
${ }^{22}$ Bauch (1923b, 304, vgl. 308 u. ö.) zufolge bleibt der Kant der Kritik der reinen Vernunft noch zu sehr der „Isolierung“ der Form gegen $\neg$ über dem Inhalt verhaftet. Die Formel vom ,Gewühl von Empfin ᄀdungen` brandmarkt er als leere Abstraktion (1923b, 259; 1926, 203, 243). (Kant selbst spricht in der ersten Kritik (KrV A 111) von einem „Gewühl von Erscheinungen“.)
} 
los" erscheinen und die Behauptung der Notwendigkeit eines vermittelnden Dritten, d. i. des transzendentalen Schemas, als grundlos. 23

Zwar redet Bauch in seiner Deutung vorwiegend von einer Vermittlung zwischen Kategorie und Anschauung, während es Kant um eine Anwendung der Kategorie auf die Erscheinung geht, gleichwohl ist die Stoßrichtung klar: Bauch will die ursprüngliche Heterogenität überwinden zugunsten eines ursprünglichen Zusammenhangs im Begriff der Erkenntnis; folglich wird die interne Geltungsgesetzlichkeit dieser Einheit expliziert. Deshalb bedarf es keines Schemas. Bei der Diskussion von Bauchs Bewältigung des Kantischen Anwendungsproblems, d. i. des Problems der konkreten Gegenstandsbestimmung, wird deutlich, daß, mit Blick auf Kants Schematismus formuliert, die Empfindung der Qualität zum „Bauelement“ des Anschauungsgegenstandes durch den Begriff wird, der den Gegenstand als kategoriales Gefüge selbst zur Einheit der Mannigfaltigkeit seiner Elemente fügt: die Empfindung ist, um Empfindung zu sein, selbst immer schon eingebettet in den allgemeinen kategorialen Zusammenhang (und also nicht eine bloße Zustandsmodifikation des Subjekts, bloß Subjektives, sondern, um solches sein zu können, Objektives). ${ }^{24}$
Am „positiven Sinn“ des Schemas, d. i. seiner berechtigten Geltungsfunktion, will Bauch „ohne ,Drittes“" festhalten, macht doch schon die Kategorie die Einheit von Kategorie und Anschauung aus (1923b, 237). Das Schema ist somit ebenfalls kategorial bedingt. Als dessen positiver Sinn bleibt, daß es, wie Kant sagt, „eine Regel der Bestimmung unserer Anschauung gemäß einem gewissen allgemeinen Begriffe" (KrV B 180) ist, eine „Methode“ oder „Verfahren“, einem „Begriff sein Bild zu verschaffen“ (KrV B 179 f.): die Anschauung wird durch den Begriff bestimmt. In dieser Bestimmung der Anschauung durch das Schema, das die Kategorie sich gibt, um sich mit dem Schema ein Bild zu geben, d. h. die Anschauung zu bestimmen, liegt für Bauch (1923a, 239) „der wahre und gute Sinn des Schemas". Das Schema macht somit die grundlegende Einheitsbeziehung der Kategorie auf die Bestimmung der Anschauung aus; die Kategorien blieben sonst Bestimmungsfunktionen, ohne einen Gegenstand vorstellen zu können. In der wirklichen Erkenntnis sind Kategorie und Anschauung, was sie sind, nur in bezug aufeinander (1923a, 240). Soweit der ursprungstheoretische Sachverhalt bei Bauch. Er weist freilich schon auf den bestimmungslogischen Sachverhalt konkreter Gegenstandsbestimmung hin. Darauf komme ich im vi-

\footnotetext{
${ }^{23}$ Bauch verweist dabei auch auf die Arbeit von Curtius.

${ }^{24}$ Vgl. Bauch 1923b, 275. Vgl. dazu ebenfalls Bauchs Unterscheidung von Qualitätsempfinden und Empfindungsqualität (1923b, 251).
} 
erten Teil zurück.

(2) Für Cohn jedenfalls liegt es ursprungstheoretisch nicht anders. Cohns Lehre vom Utraquismus ist der beredte Zeuge dafür.25 Der Utraquismus betrifft den Zusammenhang letzter Momente aller Erkenntnis: Form und Inhalt des Denkens, Denkform und Denkinhalt. Der Inhalt ist Cohn zufolge insofern zwar ,denkfremd', als er aus dem Denken nicht abgeleitet werden kann, sondern gegeben ist; um denkbar zu sein aber, müsse dem Denkfremden ,Form' (des Gegebenseins) eignen; nur so sei es erkenntnisdifferent, trete es in das Gebiet des Denkens ein. Die Letztmomente alles Erkennbaren seien nur in wechselseitiger Korrelation denkbar: ein ungeformter Inhalt könne ebensowenig in das Gebiet des Denkens eintreten wie eine gänzlich inhaltslose Form. Entsprechend wendet auch Cohn sich gegen einen fixen, nicht utraquistisch gedachten Gegensatz von Anschauung und Begriff (1923b, 255) bzw. sieht im Gegensatz von Sinnlichkeit und Verstand eine „ziemlich primitive Psychologie", die Vermögensund Wertbegriffe unheilsam verquicke (1908, 99). Kants Diktum, „Gedanken ohne Inhalt sind leer, Anschauungen ohne Begriffe sind blind" (KrV B 75), wird also wie bei Bauch sozusagen utraquistisch weitergebildet, $d$. h. in seinem notwendigen erkenntnisfunktio- nalen Zusammenhang konzipiert anstatt auf eine nachträglich zu überwindende Entgegensetzung reduziert: wie für Bauch gibt es für Cohn in der Erkenntnis weder leere Gedanken noch blinde Anschauungen $(1908,117)$.

(3) Lasks Urteilslehre stellt die einzig ernst zu nehmende Ausnahme im Lager der Südwestdeutschen dar. Bauch (1923b, 192 ff., 200 ff.), Cohn (1923b, 153 f.) und Rickert (1928, 283 f., 335 f., vgl. 278-297, 332 ff.) kritisieren sie unisono als nicht radikal genug. Im ausdrücklichen Anschluß an Kant (Lask 1911, 73 f., 80; vgl. 1912, 328 ff.) führt Lask nämlich einen Sachverhalt ein, den es für die Kritiker logisch gar nicht gibt: ein dem Logischen gegenüber schlechthin Unabhängiges, „Gegebenes“, eine „logisch amorphe Materialmasse“, die als "logisch Nacktes“ und „materiales Substrat" von der Erkenntnis mit kategorialen Prädikaten als dem „bloß logischen Zusatz“ ausgestattet und so in kategoriale Bestimmungen "hineingestellt" wird (1912, 333; vgl. 1911, 73 ff.). Denkfremdheit in diesem Sinne ist gemäß den Prinzipien des Denkens jedoch ausgeschlossen. Der Ursprung geriete zu einem hypostatischen Dualismus von Kategorie und Material, das es „unabhängig von kategorialer Um $\neg$ schlossenheit", eben „kategorial unbetroffen“ geben soll. Indes enthält das Material als Material der

\footnotetext{
${ }^{25}$ Vgl. zum Utraquismus vor allem: Cohn 1923b, 130 ff. Vgl. auch: Cohn 1908, 116 ff.; 1923a, 9 f.; 1932 , 36 f. Vgl. dazu Krijnen (2008b, Kap. 5).
} 
Erkenntnis selbst immer schon Kategorien. Lask fingiert also letzte fixe Bausteine der Erkenntnis. Inhalt ist selbst ein logisches Prinzip: von der Geltung unabhängiges, „unbetroffenes“, „geltungsfremdes" Material gibt es für die Erkenntnis nicht. Wie Rickert für die südwestdeutschen Neukantianer maßgebend herausgearbeitet hat, besteht das Minimum des Denkbaren aus Form und Inhalt, aus Logischem und Alogischem. Es wundert also nicht, daß man gerade in Lasks Geltungstheorie eine positive Aufnahme des Kantischen Lehrstücks vom Schematismus erblickt hat. ${ }^{26}$ - Lasks Letztbegründungskonzept bietet allerdings auch Ansätze für eine versöhnlichere Lesart, die ihn freilich gänzlich an die Einheitstendenz der südwestdeutschen Schule rückbindet.27 Den Schlüssel dazu bildet Lasks Begriff der ,gegenständlichen Strukturform“ qua "Urstruktur“, deren funktionale Glieder „Form und Material“ sind $(1911,281) .28$ Lask bezieht sich dabei ausdrücklich auf Rickerts Lehre.

(4) Ebenjene Aufeinanderbezogenheit der Glieder des Ursprungsverhältnisses hat Rickert mittels seines ,Gegenstandsmodells' in aller Klarheit und für die Südwestdeutschen maßgebend herausgearbeitet. Die aufgezeigte Fortbildungstendenz des Kantischen Fundamentaldualismus der Erkenntnis bei Bauch und Cohn kulminiert sachlich in Rickerts Gegenstandsmodell. ${ }^{29}$ Es ist ein Modell, das den Ursprung der Erkenntnis, den Theoretisches schlechthin fundierenden und in jedweder Konkretionsbestimmung vorausgesetzten sachlichen Bestand begrifflich bestimmt: im Ursprung wird theoretische Gegenständlichkeit und damit die Erkenntnisrelation selbst konstituiert.30 Kants Stämme der Erkenntnis werden also hinsichtlich ihrer primärkonstitutiven Bedeutung in das Gegenstandsmodell integriert. Entsprechend etabliert das Gegenstandsmodell zugleich ein Substrat möglicher Bestimmungen der Erkenntnis. Der Inhalt erweist sich als der ,logische Ort für das Alogische' und als solcher als das zu bestimmende Bestimmbare. Indem der Ursprung die Möglichkeit jeglicher Bestimmung von Gegenständen selbst ermöglicht, betrifft die logische Ursprungssphäre nicht das Denken in seiner Bestimmung von Gegenständen

\footnotetext{
${ }^{26}$ So bei Nachtsheim (1992, 180-87).

${ }^{27}$ Vgl. für eine solche Lesart die Analyse von Nachtsheim (1992, 231 ff.), die er neuerdings modifiziert und weitergeführt hat (Nachtsheim 2017). In dieser neuesten Arbeit wird deutlich sichtbar, wie sehr Lask der Heterologie der südwestdeutschen Schule verbunden bleiben möchte.

${ }^{28}$ Es handelt sich um eine aus dem Nachlaß herausgegebene Notiz, die Lask ihrem Sinn nach als „gegenständliche Urstruktur“ oder eben „gegenständliche Strukturform“ in seine Lehre vom Urteil eingearbeitet hat (Lask 1912, 364 ff., 381 ff.). Dennoch bedient Lask sich immer wieder einer hypostasierenden Rede von ,Kategorie - Material', als gebe es beide außerhalb der Struktureinheit und würden sie erst nachträglich in der Erkenntnis zusammengebracht.

${ }^{29}$ Vgl. zu diesem Modell ausführlich Krijnen (2001, Kap. 5).

${ }^{30}$ Insofern denke ich nicht, daß Rickerts Gegenstandsmodell ein „Analogon“ (Nachtsheim 1992, 186, Anm. 28) bietet für das Problem der Synthesis von Form und Inhalt im empirischen Gegenstand, das in Kants Schematismuslehre thematisch ist.
} 
unmittelbar, sondern rein im Ansatz. ${ }^{31}$ Seiner fundierenden Funktion gemäß ist der Anfang allerdings zugleich Anfang des Fortgangs: er enthält ebenfalls die Grundbestimmtheit der Bestimmungssphäre.

In Rickerts „Modell eines theoretisch gedachten Gegenstandes überhaupt" ist der theoretische Gegenstand überhaupt thematisch qua Inbegriff logischer Fundamentalbedingungen alles wahrheitsreferenten Denkens und aller wahrheitsreferenten Gedanken (Rickert 1924, 10; vgl. 1921, 50 ff.). Die Bestimmung desjenigen Gedankens, der allen anderen logisch (,begrifflich') vorausgeht und insofern sachlicher Anfang des Denkens ist, entzündet sich bei Rickert an der Frage: „Wie denken wir überhaupt etwas so, daß es Gegenstand der Erkenntnis wird? Oder: wie denken wir theore $\neg$ tisch, und was ist ein theoretisch gedachter Gegenstand?" Deren Beantwortung führt zum Begriff des ,rein Logischen' oder auch: des ,rein Theore $\neg$ tischen' als dem „rein logischen Gegenstand“, des „gedachten Etwas überhaupt" (1921, 50 f.; 1924, 8 f.), des "logischen Urphänomens" (1921, 61; 1924, 25). Ist das Denken philosophischer Gegenstand hinsichtlich des von „vornherein Gegenstän- dlichen“, des „Gegenstandes des Denkens", dann ist es thematisch hinsichtlich seiner Gehaltlichkeit. Zum Begriff dessen, was gedacht wird, wenn etwas überhaupt theoretisch oder logisch gedacht wird, gehören Rickert zufolge Form und Inhalt. Dieser Inhalt ist kein besonderer (dieser, jener) Inhalt, sondern "Inhalt überhaupt“; er muß daher ebenfalls formal genannt werden: Inhalt ist selbst Bestandteil des Gegenstandmodells, gehört also zu den formalen Faktoren des theoretischen Gegenstandes überhaupt (1921, 52 f.; 1924, 11 ff.). Damit erhält ,Inhalt' den Rang eines Prinzips; Inhalt ist selbst eine Form logischen Denkens: das Denken setzt von sich aus das, worauf es bezogen ist, involviert eine Selbstbeziehung auf Inhalt. ,Inhalt überhaupt ist der ,logische Ort für das Alogische (Rickert 1921, 52; 1924, 12). Als logischer Ort für das Alogische garantiert er die Inhaltsbezogenheit des Denkens und ermöglicht dadurch eine Mannigfaltigkeit von Inhalten. Entsprechend fungiert das Denken als Umgreifungseinheit, innerhalb welcher die Glieder der Einheit selbst möglich sind: sie haben ihren Sinn nicht als ein ,Seinsverhältnis', als gäbe es zwei reale Entitäten außerhalb der Erkenntnisrelation, die quasi nachträglich eine Er-

\footnotetext{
${ }^{31}$ Rickerts ,Etwas überhaupt' des Gegenstandsmodells darf noch nicht als ,bestimmtes Sein' aufgefaßt werden, allenfalls als bestimmbares: es liegt der Sphäre von Bestimmtheit noch voraus. In der Terminologie der Logik des Prädikats gesprochen, enthält das „Etwas über $\neg$ haupt' noch keine ,Erkenntnisformen', sondern nur ,Denkformen': nur Formen des Subjektbegriffs, nicht des Prädikatbegriffs (Rickert 1930, 111 ff.). Es liegt somit allenfalls eine Setzungsleistung von zu Bestimmendem vor; als Subjekt möglicher Prädikate ist das Etwas noch unbestimmt. In der Bestimmungssphäre rückt der logische Gegenstand des Ursprungs an die Stelle des Subjektbegriffs im Urteil. Vgl. zur Thematik näher Krijnen (2008b, Kap. 2).
} 
kenntnisbeziehung eingingen. Vielmehr, jedweder Ontik begrifflich vorausliegend, gibt es die Relata nur innerhalb der Erkenntniseinheit: sie sind analytische Derivate, Geltungsmomente dieser Einheit.

Im Horizont des Denkens gibt es nichts radikal Denkfremdes mehr, keine radikale Heterogenität von Opposita, die einer nachträglichen Vermittlung bedürften. Form und Inhalt etwa sind begrifflich isolierte Momente des Denkens. Die Form hat nicht mehr das, wovon sie Form sein soll, außer sich, sondern das Verhältnis von Form und Inhalt der Erkenntnis ist geradezu selbst ein Formverhältnis. Nur innerhalb der theoretischen Sphäre kann von einem (theoretischen) Inhalt die Rede sein. Beide sind gleichursprüngliche Glieder der Erkenntnisrelation, die trotz ihrer Verschiedenheit in begrifflich unlösbarer Verbundenheit stehen. Die Form ,Inhalt überhaupt' ist der Geltungsgrund dafür, daß es überhaupt einen Inhalt im theoretischen Verstande gibt: Inhalt ist dem Denken prinzipiell zugeordnet. Es wird also kein Alogisches in das reine Denken aufgenommen, sondern Inhalt ist selbst ein logisches Prinzip: der ,logische Ort für das Alogische'. Alles, was in das Denken eingehen kann, ist daher vom Denken konstituiert, und damit zuletzt von ihm selbst gegeben: nur aufgrund seiner eigenen Prinzipien kann das Denken $\mathrm{zu}$ irgendeinem Inhalt kommen. Nur innerhalb der Erkenntnisrelation macht die Rede von ,Form', ,Inhalt' $u$. dgl. Sinn. Weit entfernt von NichtGeltungsartigem, von etwas nicht aus dem Denken Konstituiertem, gibt es bestenfalls Stufen relativer Ursprünglichkeit (Stufen von Apriorität).

Dieser Gedanke relativer Ursprünglichkeit oder gestufter Apriorität bietet sodann den Schlüssel für die Lösung jenes Problems, das Kant mit seiner Schematismuslehre der Kritik der reinen Vernunft zu lösen versucht. Dieser Sachverhalt wird im vierten Teil dieser Studie zunächst anhand von Rickert und Bauch dargelegt und anschließend im fünften Teil in bezug auf die gegenwärtige Transzendentalphilosophie weiter ausgeführt. Bislang wurde jedenfalls sichtbar, daß Südwestdeutsche wie Rickert, Bauch oder Cohn nicht versuchen, das Material der Erkenntnis aus der Form der Erkenntnis, herauszuklauben', sondern darauf insistieren, daß schon dem Inhalt als Inhalt Form $\neg$ bestimmtheit eignet: ,Inhalt', ,es gibt', 'Sein', ,Material' usw. sind selbst Formbestimmungen, ohne die Konkretes, das immer aus Form und Inhalt besteht, geformter Inhalt durch formende Form ist, nicht einmal als ,Material' bestimmbar wäre. Statt eines schroff $\neg$ en Dualismus' von Form und Inhalt fordert die intragnoseologische Wende im Denken über den ,Gegenstand der Erkenntnis', ,Inhalt' (,Sein', ,Material' usw.) als Form in seiner Bezogenheit auf den ,Inhalt des Inhalts' zu verstehen. Diesen kön- 
nen wir, wie der Neukantianer Rickert sagt, nur „,erleben' oder ,schauen“ oder sonst irgendwie alogisch erfassen" (1921, 53 f.; 1924, 13, 15). Gäbe es nur Rezeptivität, es gäbe weder Erkenntnis noch Gegenstände der Erkenntnis: wir kämen über den bloßen ,Erlebnisstrom' nicht hinaus. Primär-konstitutiv gesehen, ermöglicht die Form ,Inhalt überhaupt', daß überhaupt Inhalt als $\mathrm{zu}$ Objektivierendes in die theoretische Grundrelation eingeht, Inhalt also theoretisch different, theoretisches Problem wird.

Da jeder Inhalt geformter Inhalt und jede Form inhaltserfüllte Form ist, gibt es in einer isolierten Betrachtung keine adäquate Bezeichnung für einen von der Form ,unberührten' Inhalt, der als „,rein' alogischer Inhalt" aufträte: dergleichen wäre nicht mehr logisch oder theoretisch qualifizierbar; die Rede davon ist deshalb logisch gesehen unverständlich und die vermeintliche Sache theoretisch irrelevant 32 Das Gegebene kann nie vom Denken losgelöst und verselbständigt werden; es ist immer nur dem Denken gegeben; nur innerhalb der theoretischen Sphäre, des Denkens hat die Rede von einem ,Gegebenen' Sinn. Die völlige Isolation von Form bzw. Denken und Inhalt ist ein Residuum der direkt-gegenständlichen Auffassung. Indes hat das Form-InhaltGefüge jenseits allen Ontizismus bei den Südwestdeutschen einen rein ,semantischen' (Zocher) oder ,funktionalen' (Bauch, Cassirer) Sinn.

\section{Die südwestdeutsche Alternative für Kants Schematismus: Methodolo- gie}

(1) Im Ergebnis ist bislang eine radikale Heterogenität im Ursprung abgewehrt und ein Verhältnis von aufeinander notwendigerweise bezogenen Gliedern etabliert. Kants Problem des Schematismus betrifft jedoch nicht das allgemeine Verhältnis von Anschauung und Begriff bzw. Rezeptivität und Verstand, sondern das viel speziellere der ,Anwendung der Kategorien auf Gegenstände der Erkenntnis', d. h., thematisch ist das Problem konkreter Gegenstandsbestimmung. Das Konkreszenzproblem der Erkenntnis wird bei den Südwestdeutschen nicht in der Weise eines, vermittelnden Dritten' gelöst, sondern durch Explikation der ursprünglichen Erkenntniseinheit, aus dem ,Begriff', der ,Idee' der ,Form' der Erkenntnis oder wie man sich sonst auch ausdrücken mag. Es kommt somit zu einer Stufung von Prinzipien gegenständlicher Bestimmtheit. Diese Stufung reicht vom Ursprung der Erkenntnis bis hin zu seiner Vereinzelung in Konkreta. Allerdings sind an

\footnotetext{
${ }^{32}$ Vgl. dazu: Rickert 1921, 53; 1924, 13. Vgl. etwa auch Rickerts Kritik intuitio $\neg$ nistischer Erkenntnislehren (siehe dazu die Hinweise bei Krijnen (2001, Kap. 5.2.2.5) ᄀ).
} 
diesem Vereinzelungs- oder Konkreszenzgedanken eine subjektiv-logische Dimension der Aneignung des Gegenstandes durch das erkennende Subjekt und eine objektiv-logische Dimension der Bestimmtheit des Gegenstandes zu unterscheiden. Dies wird exemplarisch sichtbar durch eine nähere Betrachtung von Rickerts und Bauchs Erkenntnislehre.

Gemäß der Heterologie hat die Erkenntnistheorie an ihrem Anfang nicht die Tatsache der Getrenntheit von Form und Inhalt (Subjekt und Objekt o. ä.), sondern diese Getrenntheit erweist sich als souveränes Werk objektiven, gegenstandsbezogenen, erkenntnisinteressierten Denkens. Jedwedes denkbare Etwas besteht Rickert zufolge aus Form und Inhalt (1921, 120 f., 262)::33 ontologisch gesehen die als wahrnehmbar oder verstehbar gegebene Welt aus sinnlichen Gegenständen, denen erkenntnistheoretisch gesehen sinnlicher Inhalt und unsinnliche Form eignet, sowie unsinnlichen Gegenständen (Sinngebilden), denen erkenntnistheoretisch gesehen unsinnlicher Inhalt und unsinnliche Form zukommt (Rickert 1939a, 107 u.ö.; 1934; 1939b). Für eine Transzendentalphilosophie auf der Reflexionsstufe Rickerts kann es nichts Denkfremdes, nichts Nicht-Geltungsartiges, nichts nicht aus der Geltung selbst Konstituiertes ge- ben, sondern höchstens, wie angedeutet, Stufen relativer Ursprünglichkeit innerhalb der theoretischen Sphäre. Freilich muß ein Inhalt, da' sein, der erkannt wird, aber sogar ,Inhalt' entpuppte sich als Prinzip innerhalb der Geltung. ,Da' ist etwas nur durch den Gedanken - die Geltungsstruktur des Gedankens ist aller Erkenntnis von Seiendem in seinem Dasein und Sosein logisch vorgängig. Die Stufen relativer Ursprünglichkeit innerhalb der theoretischen Sphäre reichen bei Rickert von der Ursprungssynthesis des Denkens im Gegenstandsmodell bis hin zur Konstitution des konkretgegenständlichen Sinns durch methodologische Erkenntnisformen. Decken die Stufen relativer Ursprünglichkeit das gesamte Bestimmungsspektrum der theoretischen Sphäre ab, dann muß auch der Begriff der Wirklichkeit und der des Gegebenen der Erkenntnis konstituiert werden. Nicht die Inhaltsbezogenheit des Denkens selbst steht dann zur Debatte, sondern der spezifische Sinn des als Material (etwa der Einzelwissenschaften) Gegebenen d. h. Gegebenes einer relativ späten Fundierungsstufe. Die Erkenntnisbeziehung wird somit prinzipientheoretisch konkretisiert: begrifflich verdichtet zu konkret-gegenständlichem Sinn.

Bezüglich Rickerts Philosophie gilt es dabei sich der Eigentümlichkeit

\footnotetext{
${ }^{33}$ Darin steckt freilich ebenfalls eine wichtige Differenz zu Kants transzendentaler Ästhetik der Kritik der reinen Vernunft, §§ 2 ff., denn Raum und Zeit können nicht als Primärformen des unmittelbar Gegebenen fungieren. Vgl. dazu Krijnen (2013).
} 
zu vergegenwärtigen, daß Rickert das Problem der Gegenstandskonstitution in zweierlei Richtung ausgelotet hat: in eine subjektiv-logische und eine objektiv-logische Richtung. 34 Rickerts erkenntnistheoretisches Hauptwerk Der Gegenstand der Erkenntnis ist subjektiv-logisch ausgerichtet; erst im Laufe mehrerer Überarbeitungen hat er es auch objektiv-logisch konturiert und entsprechend objektiv-logische Aspekte eingebaut. ${ }^{35}$ Da Rickert einen logischen Primat des objektiven Weges vertritt, handelt es sich weniger um zwei Wege der Erkenntnistheorie als vielmehr um die Erkundung zweier (heterologisch vermittelter) Dimensionen oder Sphären der Erkenntnis, also um zwei Reflexionsweisen der Erkenntnistheorie. Diese thematisiert als Lehre von der Geltungsbestimmtheit der Erkenntnis immer den Einen, Gegenstand der Erkenntnis' qua Maßstab. Dies erfolgt jedoch in zwei Hinsichten. Zum einen geht es um die Erkenntnis qua ,Gegenstand der Erkenntnis', um das ,gedachte Etwas', das, was gedacht wird als objektives Gebilde, als Objekt: um die Objektivität selbst der Erkenntnis. Diese betrifft die Geltung als Inbegriff von Prinzipien, welche die Objektivität jeweiliger Erkenntnisleistungen garantieren. Während auf objektivem Weg der ,Gegenstand' für sich und damit losgelöst vom erkennenden Subjekt gedacht ist, geht es, zum anderen, beim subjektiven Weg um die Erkenntnis qua ,Erkenntnis des Gegenstandes', um die Erkenntnis durch ein Subjekt: um die Subjektivität der Erkenntnis. Diese betrifft die Geltung der Erkenntnis in ihrem logischen Vollzug. Entsprechend ist im Ganzen der Erkenntnis das, gedachte Etwas', das, was gedacht wird als objektives Gebilde, logisch zu unterscheiden vom ,Akt des Denkens', vom Wodurch des Denkens als subjektivem Gebilde.

Diese zwei verschiedenen Aufgabenstellungen der Erkenntnistheorie führen nicht nur $\mathrm{zu}$ je unterschiedlichen Erkenntnisbestimmungen; sie sind zudem mit zwei verschiedenen Ausgangspunkten der Geltungsreflexion verbunden: Die Erkenntnistheorie geht immer von einem ,Faktum', einem Wirklichen aus (Rickert 1909, 174, 181, $189,193 ; 1928,251 ; 1930,25)$. Rickerts subjektiver, auch ,transcendentalpsychologisch' genannter Weg geht vom Faktum des Erkennens aus: vom „wirklichen Erkenntnisakt“. Vom subjektiven Akt ausgehend, bahnt sich die Erkenntnistheorie „allmählich" ihren Weg zum transzendenten Gegenstand als Grund aller Objektivität (1909, 174): sie dringt vom „Subjekt zum Objekt" vor $(1928,4)$. Der objektive, auch

\footnotetext{
${ }^{34}$ Vgl. zu dieser Thematik Rickert (1909, 1912; 1928). Dazu vgl. Krijnen (2001, spez. Kap. 6; 2014a; 2014b).

${ }^{35}$ Rickert $(1928$, X) bezeichnet die 3. Auflage von 1915 sogar in gewisser Hinsicht als ein „neues Buch“; er rät vom Gebrauch der früheren Auflagen ab, da so gut wie kein Abschnitt wörtlich derselbe geblieben sei. Vor allem (jedoch nicht nur) hat er seine Abhandlung über die „Zwei Wege der Erkenntnistheorie“ (Rickert 1909) eingearbeitet und damit den objektiven Weg.
} 
,transcendentallogisch' genannte Weg Rickerts behandelt „so schnell wie möglich“ und "ohne Rücksicht auf den psychischen Akt des Erkennens" den transzendenten Gegenstand in sich: er geht „,rein' logisch“ vor $(1909,174)$. Anders als im subjektiven Verfahren läßt das objektive die Frage nach der Erkenntnis des Gegenstandes zurücktreten. Daher fungiert als Ausgangspunkt nicht das subjektiv-logische Faktum des Erkennens, sondern das objektivlogische der Erkenntnis: der "wahre Satz" (1928, 254 mit 251; 1930, 5 f.).

Im 5. Kapitel seines Gegenstandsbuches bietet Rickert eine Auseinandersetzung mit dem sog. empirischen Realismus, in der er versucht zu zeigen, $\mathrm{da} ß$ und wie der transzendentale Idealismus mit dem empirischen Realismus (der Einzelwissenschaften) verträglich ist (vgl. Krijnen 2014b). Dies ist insofern interessant, als hier systematisch gesehen das Konkrezenzproblem der Erkenntnis, das Kant im Schematismuskapitel umtreibt, subjektiv-logisch thematisch ist. 36

Aus subjektiv-logischer Sicht sind Stufen der Aneignung des Gegenstandes durch das Subjekt thematisch. Der Geltungsgrund der Erkenntnis, der ,Gegenstand' qua Maßstab der Erkenntnis hat sich dabei als ein "Sollen“, nicht als ein „real Seiendes“ erwiesen (Ric- kert 1928, 350): das Subjekt ist hinsichtlich der Geltung seiner Leistungen einer es normierenden Instanz unterworfen. Seiner Auffassung der Erkenntnistheorie als Formenlehre entsprechend, bezieht sich Rickerts Analyse auch im 5. Kapitel auf die „Form“ (Rickert 1928, 361) der Erkenntnis und damit auf die Geltungsprinzipien, die der Erkenntnis zugrunde liegen. Näherhin handelt es sich im 5. Kapitel um eine spezifische Form, die Rickert als „Kategorie“ bezeichnet: durch sie wird „unser Erkennen gegenständlich“ (1928, 361, 366). Dieses Gegenständlichwerden der Erkenntnis durch die Kategorie wird gemäß der Anlage des Gegenstandsbuches subjektiv-logisch und daher in der Perspektive des Gegenständlichwerdens des Erkennens eruiert (1928, 362 ff.).

Es erweist sich als ein komplexer Sachverhalt, der aus einer Vielzahl mit je spezifischer Erkenntnisfunktion für das Erfassen des Gegenstandes durch den Erkenntnisakt ausgestatteten Formen besteht (Rickert 1928, 365 ff.). Was in subjektiv-logischer Betrachtung speziell die „Kategorie“ ausmacht, ist, daß durch sie sozusagen der „Übergang vom Sollen zum real Seienden" vollzogen wird, d. h., Rickerts Kategorie ist jenes Moment im Erkennen, das dem Inhalt die ihm zugehörige Form „bei-

\footnotetext{
${ }^{36}$ Rickerts Gegenstandsbuch liefert kein alle Prinzipienstufen der Erkenntnis umfassendes System der Erkenntnis, sondern ist auf das erkenntnistheoretische Problem der Wirklichkeitserkenntnis abgestellt. Das Problem der ursprünglichen Synthesis der Erkenntnis wird hier nicht traktiert. Rickert diskutiert es im Kontext seiner ,Heterologie'. Dabei geht er jedoch objektiv-logisch vor und nicht, wie im Gegenstandsbuch, subjektiv-logisch. Dadurch ergibt sich ein anderes, nämlich ein objektiv-logisches Stufenmodell.
} 
legt", etymologisch: von ihm „aussagt" $(1928,366)$. Sie ist Aussage- oder Beilegungsform, Form der „Prädizierung“, und damit diejenige Form des Sinnes, die der "Akt der Anerkennung" und folglich das Erfassen des Gegenstandes durch das erkennende Subjekt hat (1928, 355 f.). Daß die Geltung der Erkenntnis in der Kategorie qua Form der Anerkennung theoretischer Normativität „begründet“ $(1928,369)$ ist, wird subjektiv-logisch so geklärt, daß Erkenntnis als ein Formverleihungsprozeß dargestellt wird. Die Übereinstimmung von Denken und Wirklichkeit wird entsprechend nicht realistisch (extragnoseologisch), sondern intragnoseologisch gedeutet. Diese intragnoseologische Deutung führt zu einer subjektiv-logischen Konstitutionsordnung, zu einem Inbegriff von Formen der Erkenntnis, der subjektiv-logisch sichtbar macht, was die Erkenntnis „Voraussetzt und voraussetzen muß, um Anspruch auf Objektivität zu erheben" $(1928,369)$.

Damit ist im Grunde das Gegenständlichwerden der Erkenntnis (subjektivlogisch) abgehandelt. Rickert erörtert anschließend Spezialprobleme, die die Verträglichkeit von transzendentalem Idealismus und empirischem Realismus einleuchtend machen sollen. An diesen Erörterungen ist für die Idee einer subjektiv-logischen Erkenntnisbegründung und die darin liegende Versöhnung von Erkenntnisformen und Wirklichkeit in der konkreten Erkennt- nis Folgendes von Interesse:

Das erste Spezialproblem betrifft das der "Gegebenheit"; Rickert faßt diese als „Kategorie“ und damit als eine subjektiv-logische Größe (1928, 371 f.). Freilich geht es in diesem Kontext nicht mehr um die (schon erfolgte) erkenntnistheoretische Zurückweisung einer empiristischen Basis- oder Rezeptionstheorie, sondern um den Sinn konkret-gegenständlicher empirischer Erkenntnis in puncto Gegebenheit. Entsprechend klärt Rickert subjektivlogisch, was es mit der Form der Gegebenheit oder Tatsächlichkeit für die empirische Erkenntnis auf sich hat. Es wird gezeigt, daß die Geltung von Tatsächlichkeits- oder Gegebenheitsurteilen insofern in der Form gründet, als die Kategorie qua Form des Erkenntnisaktes ihnen erst "Gegenständlichkeit“ verleiht $(1928,372)$. Das tatsächlich Gegebene erweist sich also als formbestimmt. An die Stelle eines Gegensatzes von tatsächlich Gegebenem bzw. „Erfahrung" und „Denken“ tritt das Denken als formale Grundlage auch der Wirklichkeitserkenntnis (1928, 378, 381).

Diese Reduktion des empirischen Realismus auf die Tatsachenproblematik reicht freilich nicht aus, um die empirische Erkenntnis zu verstehen. Deren Sinn enthält eine Reihe weiterer Voraussetzungen, die weit über das tatsächlich Gegebene hinausgehen, vor allem diese, daß Tatsachen immer Teil eines größeren Zusammenhangs sind. 
Gerade wenn man die wissenschaftliche Erkenntnis als Paradigma nimmt, läßt sich nicht übersehen, daß sie auf „in sich zusammenhängende Erkenntnis" aus ist (Rickert 1928, 383). Dieses Streben nach zusammenhängender Erkenntnis des Wirklichen hat ebenfalls ein subjektiv-logisches Fundament. Da jedwede Wissenschaft Erkenntnismaterial bearbeitet, lassen sich am wissenschaftlichen Erkenntnisanspruch näherhin unterscheiden: 1. das Material und 2. dessen Bearbeitung. Zunächst (§ IV) weist Rickert die Vereinbarkeit von transzendentalem Idealismus und empirischem Realismus für das Material nach, anschließend ( $\S$ V) für die Bearbeitung. Dabei ist so etwas wie „Material“ freilich schon ein recht weit konstituierter Gegenstand, also eine Konkretion von Form-InhaltVerhältnissen. Und freilich gelten die dargelegten Verhältnisse von Material und Bearbeitung des Materials ganz allgemein für die Erkenntnis, nicht bloß für die wissenschaftliche.

Die Kompatibilität von Denken und Wirklichkeit läuft in diesem Problemkontext auf den Nachweis hinaus, daß auch das vom erkennenden Subjekt vorausgesetzte Material des Erkennens als zusammenhängende reale Welt transzendental-idealistisch gesehen „Formen“ des Zusammenhangs enthält (Rickert 1928, 389 f.). Subjektiv-logisch betrachtet ist Erkennen ein SubjektObjekt-Verhältnis, und es sind die Akte des Subjekts, durch die es erkennt. Der
Gegenstand qua Maßstab des Erkennens erweist sich sodann als ein sollensnotwendiges Zusammen von Form und Inhalt, während durch die Kategorie der vom erkennenden Subjekt als fertig vorausgesetzte Gegenstand erst logisch ,produziert' wird $(1928,391)$. Dieser subjektiv-logische Sachverhalt ist das Entscheidende für Rickerts Behandlung der sog. "Wirklichkeitsurteile“ (1928, Kap. 5, § IV). Nicht auf den Inbegriff der Wirklichkeitsformen, sondern auf die durch die Kategorie(n) vermittelte Gegründetheit im Sollen als Gegenstand kommt es an. Immer bildet die Kategorie den „Übergang vom Sollen zum wirklich Seienden", hier: die Kategorien des Zusammenhangs (1928, 393).

Rickert führt seinen transzendentalidealistischen Erkenntnisbegriff also immer weiter aus. Auch die ,empirischrealistische' Voraussetzung einer objektiven Wirklichkeit erweist sich erkenntnistheoretisch als Ergebnis kategorialer Anerkennung. Erkennen bleibt durchgehend ein Anerkennen von Normen, die als Maßstab auch der Wirklichkeitserkenntnis fungieren (Rickert 1928, 394 f.). Näherhin handelt es sich um Anerkennungsformen des „erkenntnistheoretischen Subjekts": dieses bringt die objektive Wirklichkeit subjektiv-logisch zustande, und damit das, was empirisch-realistisch gesehen die für sich bestehende objektive Wirklichkeit ist (1928, 397 f.). Zugleich wird verständlich, inwiefern es für das 
reale, „erkennende Subjekt" eine von ihm unabhängige objektive Wirklichkeit gibt; denn diesem erkennenden Subjekt ist das erkenntnistheoretische Subjekt die Norm oder das "Ideal“ (1928, 397 ff.). Gemäß Rickerts Idealismus ist die objektive Wirklichkeit geradezu bestimmt als von „aller wissenschaftlichen wie vorwissenschaftlichen Begriffsbildung eines realen Subjekts freie Wirklichkeit" (1928, 414). Als solche ist sie immer schon formbestimmt.

Damit ist aus subjektiv-logischer Sicht das Problem der objektiven Wirklichkeit jedenfalls programmatisch gelöst. In $\S \mathrm{V}$ müssen entsprechend diejenigen Formen bestimmt werden, die die begriffliche Bestimmung der objektiven Wirklichkeit durch das erkennende Subjekt leiten. Sie machen, sofern es sich um wissenschaftliche Erkenntnis handelt, den "Begriff der Wissenschaft vom realen Sein" aus (Rickert 1928, 402): die sog. „methodologischen Erkenntnisformen". Durch sie bestimmt das erkennende Subjekt die objektive Wirklichkeit begrifflich. Diese begriffliche Bestimmung der objektiven Wirklichkeit ist subjektiv-logisch zu verstehen.

Rickert bewerkstelligt die Kompatibilität auf der Konstitutionsstufe wirklichkeitswissenschaftlicher Begriffsbildung ${ }^{37}$ Auch sie erweist sich als formbestimmt, näherhin als „umbil- dendes Auffassen" der objektiven Wirklichkeit durch das reale, erkennende Subjekt, und damit durch in ihrer Geltung von diesem Subjekt unabhängige „methodologische Formen“ (1928, 403 f.). Auch diese methodologischen Formen behandelt Rickert als Anerkennungsformen. Durch sie kommt der Sinn von Aussagen über die Wirklichkeit subjektiv-logisch zustande. Daher hängt auch die "Objektivität“ der wissenschaftlichen Begriffsbildung subjektiv-logisch daran, „ob ihre Formen in gültigen Normen begründet sind" $(1928,431)$. Erneut entstammt der Erkenntnisstoff gewissermaßen "empiristisch" den inhaltlichen Bestimmungen des tatsächlich Gegebenen, während die Gegenständlichkeit verleihende Form „idealistisch“ auf ein "Sollen“ gegründet ist: ein transzendentes Sollen fungiert als "Grundlage“ einer jedweden Erkenntnis $(1928,431)$.

Soweit die subjektiv-logische Reflexion. Rickert ist, wie angedeutet, auch den objektiven Weg in der Objektivitätsbestimmung gegangen (vgl. 1921; 1930; 1939c). Gerade die Logik des Prädikats (1930) ist ein Versuch, im Ausgang nicht vom Erkennen, sondern vom Erkannten zu zeigen, wie das Gedachte sich aufbaut (jedenfalls was die konstitutiven Momente betrifft, denn die methodologischen bleiben in dieser Schrift außen vor) 38 . Die Lo-

\footnotetext{
${ }^{37}$ Vgl. zum vorwissenschaftlichen Erkennen etwa Rickert (1929, Kap. 1.I).

${ }^{38}$ Rickert hat sie eingehend thematisiert in den Grenzen (1929) und Kulturwissenschaft und Naturwissenschaft (1926).
} 
gik des Prädikats bietet eine objektivlogische Konstitutionstheorie, die sich über „den einfachsten logischen Sinn und die Urprädikate" (Rickert 1930, 70 ff.) entwickelt.

Interessanterweise übersetzt Rickert $(1930,71,78,97)$ Prädikat im logischen

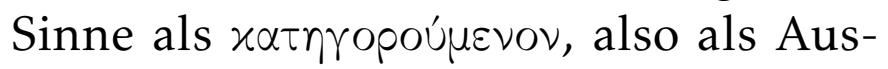
sageform. Er meint damit jedoch nicht subjektiv-logisch die Form des Aussagens, sondern objektiv-logisch die des Ausgesagten. Selbstverständlich ergibt sich auch hier eine ,logische' Fundierung jedweder, Ontologie': der transzendentale Idealismus bleibt die Grundlage eines möglichen Realismus, das Wirkliche logisch gesehen immer das als „wirklich Prädizierte“ (1930, 80, vgl. 78 ff.). Und in seiner Lehre von den Denk- und Erkenntnisformen zeigt Rickert (1930, 111 ff.), wie schon das logische Subjekt, und damit Denkbares und folglich Erkennbares, formbestimmt ist. Das im Gegenstandsbuch im Kontext der konstitutiven Wirklichkeitsformen abgehandelte ,Dies' kehrt in der Logik des Prädikat objektivlogisch zurück als unmittelbarer Bezug auf sinnlich Anschauliches, das als solches wiederum die (Denk-)Form der „Identität“ voraussetzt (1930, 114 ff., 141).

Kurzum: Die Kompatibilitätsthese von, Kantisch gesprochen, Kategorie und Erscheinung hat sich als triftig erwiesen - Erscheinungen sind auf jedweder Ebene ihrer Bestimmtheit immer schon formbestimmt. Die logis- che Abhängigkeit alles dessen, was es irgendwie gibt, von Formen des Denkens und Erkennens führt bei Rickert über die subjektiv-logische Bestimmung hinaus auch zu einer geltungsfunktionalen noematischen (objektivlogischen) Differenzierung theoretischer Geltungsprinzipien. Sie reichen von der reinen Heterogenität des Ursprungs über dessen Konkresenz im Urteil bis hin zur Konstitution von konkret-gegenständlichem Sinn durch sog. „Urprädikate“ und weitere kategoriale Formen der objektiven Wirklichkeit sowie methodologische Erkenntnisformen. Da Bauchs Erkenntnislehre in aller Konsequenz objektiv-logisch verfährt, und zwar in Auseinandersetzung mit dem Realismus wie mit Kant, will ich zum Schluß der Ausführungen zum Neukantianismus noch darlegen, wie sich hier die Verzichtbarkeit der Kantischen Schematismuslehre für das Problem der Konkreszenz der Erkenntnis darstellt.

(2) Wie für Rickert, ist für Bauch die Wirklichkeit ein eminent philosophisches Problem: was sie ist; was ihr Gegebensein bedeutet; wie sie sein muß, um erkannt werden zu können; was ihr Verhältnis zum Subjekt und dessen Denken ausmacht (Bauch 1923b, Teil I, spez. 93 ff.; 1931, 255 ff., u.ö.). Entsprechend wird ihm, wie bei Rickert, noch die ,Tatsache' zu einem erkenntnistheoretischen Problem. Wie Rickert, unterzieht Bauch die für den Realismus wichtige Unterscheidung zwischen 
dem, ,daß' etwas, und dem, ,was' etwas ist, oder einfach: Dasein und Sosein einer scharfen Kritik. Diese Kritik läuft, wie bei Rickert, darauf hinaus, daß ,Dasein' und ,Sosein' selbst in der Erkenntnis gesetzt und deren Gesetzlichkeit unterworfen sind, wenn das zu Erkennende denn Erkenntnisgegenstand soll sein können bzw. überhaupt soll sein können (Bauch 1915, 108; 1923b, 123 ff.; 1982, 262 ff.). Wie für Rickert, ist für Bauch die Realität oder Wirklichkeit selbst ein philosophisches Problem, das einer wissenschaftlichen Lösung nur transzendental-idealistisch fähig ist. Bekanntlich wehrt sich Bauch gegen die Abstraktheit des Begriffs und denkt Form und Inhalt als einen intrinsischen Zusammenhang. Stets erweist sich das Sein seiner Möglichkeit nach als im Denken begründet. Daraus ergibt sich, wie bei Rickert, ein transzendental-idealistisch transformierter Gegenstandsbegriff: Der ,Gegenstand' qua Maßstab der Erkenntnis ist Bauch keine existierende Wirklichkeit, sondern ein Inbegriff von Geltungsbeziehungen (1923b, 91 f. u.ö). Das tatsächliche, wirkliche Erkennen richtet sich nach Geltungsbeziehungen und erhält dadurch seine Gültigkeit; dem tatsächlichen Denken steht der Begriff als "Gegen-stand" gegenüber (Bauch 1923b, 217 ff.). So erweist sich auch bei Bauch, wie bei Rickert, Unwirkliches als "Grund der Wirklichkeit" (1923b, 126; 1982, 254). Nicht anders als Rickert, lehnt Bauch den Realismus als eine erkenntnistheoretische Position ab und erkennt ihn als „empirischen Realismus" an: die Grundlagen der empirischen Realität sind transzendentaler Art, Geltungsbedingungen der empirischen Realität. Und in seiner Wissenschaftslehre der Wirklichkeitswissenschaften folgt Bauch, trotz seines übergeordneten objektiv-logischen Duktus' und allerlei Präzisierungen im Detail, Rickerts Ansatz.

Damit zur objektiv-logischen Prägung der Bauchschen Erkenntnislehre und der darin liegenden Überwindung des Schematismus als eines vermittelnden Dritten. Zweifelsohne berücksichtigt Bauch auch die subjektiv-logische Dimension der Erkenntnis, integriert also, wie Rickert, beide sog. Wege in eine Erkenntnistheorie, aber insgesamt einheitlicher als Rickert und unter einem objektiv-logischen Vorzeichen. Dadurch tritt die Bedeutung von Formen der Erkenntnis als Prinzipien gegenständlicher Bestimmtheit prägnant hervor.

Für Bauch ist das Erkenntnisproblem dem Problem des Erkennens wie dem der Erkenntnis verbunden, wobei das Erkennen aus der Erkenntnis heraus $\mathrm{zu}$ verstehen, die Erkenntnislehre folglich in einer objektiven Perspektive zu entwickeln ist (Bauch 1923/24; 1923b, 49; 1982). Dies nimmt sich dergestalt aus, daß Gegenstand wie Erkenntnis gemeinsamen Bedingungen unterstehen müssen. Diese gemeinsamen Bedingungen liegen in der Wahrheit. Wäh- 
rend das Erkennen sich als Beziehen erweist, das sich in seiner Geltung oder Wahrheit nur nach Beziehungen richten kann - die somit Geltungs- oder Wahrheitsbeziehungen sind -, erweist sich auch der Gegenstand als eine durch Wahrheitsbeziehungen fundierte Beziehung. Die Wahrheitsbeziehungen, deren Inbegriff die Wahrheit ist, sind also gegenstandslogische und damit übersubjektive Beziehungen. Bauch konzipiert sie als objektive Geltungsfunktionen. Der Gegenstand ist ihm geradezu ein Stehen-in-Beziehungen und das Erkennen als ein Sich-richten-nachBeziehungen.

Näherhin erfolgt die Konstitution des Gegenstandes der Erkenntnis und die der Erkenntnis des Gegenstandes durch drei zusammengehörende Arten von Wahrheits- oder Geltungsbeziehungen: durch die Kategorie, den Begriff und die Idee.

Was die Erkenntnis wirklicher Gegenstände betrifft, geht es zunächst um die gegenstandsgebende Ordnung der Empfindungsinhalte. Um überhaupt sein bzw. Empfindung sein zu können, muß die Empfindung in einen Zusammenhang eingeordnet sein (Sein, Identität, Verschiedenheit, Inhaltlichkeit u. dgl.). Dieser Zusammenhang ist der der Kategorie. Wie bei Kant, aber anders als bei Rickert, hat bei Bauch die Kategorie einen dezidiert objektiv-gegenständlichen Sinn: Kategorien sind Prinzipien gegenständlicher Bestimmtheit, nicht Formen des Beziehens, sondern des Bezogenen. Sie sind für alles Wirkliche vorausgesetzt. Die Kategorie steht wiederum in einem Beziehungszusammenhang von Kategorien, die den Gegenstand bestimmen (Ding oder Eigenschaft, Ursache oder Wirkung, Eines oder Vieles usw.). Dieser gegenstandsbestimmende Zusammenhang von Kategorien ist bei Bauch der Begriff: das „objektive Bildungsgesetz des zu erkennenden Gegenstandes" (1982, 265) ${ }^{39}$ Im Begriff haben die Kategorien ihren Zusammenhang; zugleich sind die Gegenstände selbst durch Begriffe und damit durch Kategorien konstituiert. Der Zusammenhang der Begriffe wiederum ist die Grundlage des Zusammenhangs der Erkenntnis der Gegenstände wie der Gegenstände der Erkenntnis. Letztlich läuft diese Bauchsche Zusammenhangsphilosophie auf das Ganze des Zusammenhangs hinaus, d. h. auf die Idee als das System der Begriffe und damit als diejenige objektive Geltungsbeziehung, die als Ganzes von Bedingungen der Gegenstände die Wirklichkeit ebenso konstituiert wie deren Erkenntnis.

Geltungsbeziehungen sind Bedingungen der Gegenständlichkeit der Gegenstände, nicht selbst Gegenstände.

\footnotetext{
${ }^{39}$ Bauch bezeichnet ihn auch als Funktion des Folgeverlaufs vom Allgemeinen zum Besonderen oder als Invarianz der Bedingungen für die Varianz und Abwandlung der Besonderheiten (1923b, 283 ff.; 1926, 101 f., 131 ff., 188; 1982, 266).
} 
Sie liegen allem Seienden logisch zugrunde, und zwar von Anfang an und durchgehend. Bauch hat diesen Gedanken, wie Rickert, freilich auch hinsichtlich der Methodik konkreter Gegenstandsbestimmung qua Wirklichkeitserkenntnis verfolgt: zu den "fundamentalen Strukturformen der Wahrheit" gehört ihm auch die "Methode" als der Weg der Wahrheit (1923b, 136, vgl. Teil II und Teil III). Kurzum: bei Bauch eignet der Erkenntnis eine geltungsnoematische Struktur, die vom Ursprung von Gegenständlichkeit bis in die Bestimmtheit der Konkreta reicht und dabei als Erkenntnisbeziehung durchgehend bei sich bleibt.

\section{Zum Schematismus in der kantiani- sierenden Transzendentalphilosophie der Nachkriegszeit}

Im Grunde liegen die Verhältnisse in der kantianisierenden Transzendentalphilosophie von Hans Wagner und Werner Flach nicht anders 40 Auch hier bedarf es zur Bewältigung des Konkreszenzproblems der Erkenntnis keines Kantischen Schemas. Die Schematismuslehre Kants spielt also auch hier in puncto grundsätzlicher Fundierungsverhältnisse keine Rolle. Nachdem der diesbezügliche Grundgedanke anhand von Rickert und Bauch schon dargelegt wurde, reichen zur Klärung des Sach- verhalts einige allgemeine Andeutungen:

(1) Zunächst ist in bezug auf Wagner und Flach wichtig, daß beide ihre Systematik in der Weise einer Geltungsnoematik entwickeln, die Geltungsproblematik also unter der Ägide des ,objektiven Weges' Rickerts bewältigen. Damit verfolgen sie, systematisch gesehen, den Bauchschen Ansatz. Hinsichtlich des Kantischen Stämmedualismus kommt es dabei unter Einfluß des Neukantianismus, Husserls und Hönigswalds zu einer differenzierteren Einschätzung der Erkenntnisrelation, die sowohl geltungsnoematische als auch geltungsnoetische Aspekte integriert. So unter $\neg$ scheidet Wagner nunmehr „vier Fundamentalglieder eines theoretischen Gebildes": Subjekt - Objekt - Erkenntnistätigkeit - Erkenntnisergebnis (Wagner 1980c, 1 ff.). Flach, der Wagner hier weitgehend folgt, zeichnet ein funktionales Modell des Wissens, das ebenfalls aus vier Komponenten besteht: Intention - Aufgabe -Leistung - Gehalt (1994, 145 ff.). Obwohl sich auch bei Kant Ansätze finden, subjektive (geltungsnoetische) und objektive (geltungsnoematische) Elemente in das Modell der Erkenntnis zu integrieren, überarbeiten Wagner und Flach das zweigliedrige Modell gerade unter diesem Aspekt. Diese Überarbeitung erfolgt, wie angedeutet, im Einklang mit Kant (Krijnen 2008a, 2014a), und

${ }^{40}$ Vgl. vor allem Wagner (1980c) und Flach (1994). Siehe dazu etwa: Krijnen und Zeidler 2011; Krijnen und Zeidler 2017. 
zwar: unter der Regentschaft der geltungsnoematischen Perspektive. Wie bei Bauch rückt also die Gehaltsanalyse ins Zentrum. Von der Geltungsnoematik aus wird sodann ebenfalls die Vollzugs- und Konkreszenzproblematik der Erkenntnis aufgerollt. Was die Konkreszenzproblematik, um die es Kant im Schematismuskapitel geht, entwickelt Wagner (1980c, § 23 f.; 1992, $\S 9)$ ein Modell gestufter Apriorität, das für die Konkreszenz der Erkenntnis spezifische Prinzipien hergibt, die zur sog. regulativen und systematischen Apriorität gehören und als normierender Bestand für das erkennende Subjekt gelten. Sie ergeben sich im Zuge der geltungsnoematischen Reflexion als des „einzig möglichen Fundaments einer allgemeinen Reflexionslehre" (1980c, § 8), d. h. der prinzipientheoretischen Reflexion auf die Geltung des "Gedachten“ (Noema), nicht darauf, „daß ich denke" $\left(1992,208\right.$ f.). ${ }^{41}$
Einen Gegenstand erkennen heißt sodann, daß ihm die ihm eigenen Bestimmtheiten zugedacht werden, und zwar, wie bei Kant, in der Weise des Urteils (Wagner 1980c, § 12; 1992, 213 f.). Das Urteil ist eine Einheit von Subjektbegriff und Prädikatbegriff: Der Subjektbegriff repräsentiert den zu bestimmenden, an ihm selbst bestimmten Gegenstand; für die Erkenntnis ist der Subjektbegriff der unbestimmte noch zu bestimmende Begriff (Erkenntnisaufgabe). Der Prädikatbegriff (bzw. die Reihe der Prädikatbegriffe) indes bestimmt den Subjektbegriff und denkt damit, was der Erkenntnisgegenstand an Bestimmtheit hat. Wagner betont also, daß es sich beim Urteil um eine noematische „Bestimmungsrelation" handelt und nicht primär um „Subsumtion“:42 um die Bestimmung des Subjektbegriffs durch den Prädikatbegriff (1980c, 92 ff.; 1992, 214 ff.).

Darin liegt für die Erkenntnis, daß

\footnotetext{
${ }^{41}$ Wagner hat diesen Gedanken eingehend in Philosophie und Reflexion thematisiert (1980c, §§ 4-7), vgl. aber auch seine Auseinandersetzung mit Husserl (Wagner 1980b)) oder seinen Beitrag zur Diskussion über die Argumentationsstruktur der transzendentalen Deduktion Kants (1980a).

${ }^{42}$ In seinem Aufsatz über Kants Schematismus hebt auch Flach $(2001,38)$ hervor, daß Kants Rede von Subsumtion kein klassenlogisches Verhältnis zum Ausdruck bringen soll, sondern „Heterogenes in ein Verhältnis bringt“; die Begründung der „vereinzelten, bedingten Gegenstandsbestimmtheit durch die generelle, unbedingte Gegenstandsbestimmtheit bezieht Heterogenes aufeinander, vermittelt es miteinander". Birrer (2017, 248 f.) unterstreicht ebenfalls, daß der Subsumtionsbegriff des Schematismuskapitels nicht ein begriffslogisches Verhältnis betrifft, sondern das Zusammenhalten von Anschauung (Einzelfall) und Begriff (Regel); damit bringe Kant die "juristische Konnotation“ des Subsumtionsbegriffs in Anschlag. Indem Birrer dabei abstellt auf die Handlung des „Entscheidens“, ob ein gegebener Gegenstand der Regel entspricht, geht der systematisch entscheidende Aspekt des Bestimmens des Gegenstandes durch den Begriff und damit die Konkreszenzfunktion des Schemas jedoch unter. Der ",argumentative Beitrag“ des Schematismuskapitels besteht für Birrer entsprechend darin, die Synthesisleistung des Verstandes bezüglich einer „berechtigten Subsumtion“ zu „überprüfen“ $(2017,252)$. Dabei zitiert Birrer selbst Kants Aussage, daß die Schemata der reinen Verstandesbegriffe es ermöglichen, „Erscheinungen allgemeinen Regeln zu unterwerfen und sie dadurch zur durchgängigen Verknüpfung in einer Erfahrung schicklich zu machen" (2017, 253; vgl. KrV B 185). Überprüfung und Unterwerfung sind freilich zweierlei. Wenn ich recht sehe, kommt es daher bei Birrer (2017, 264, vgl. 264 ff.) auch zur Unterscheidung einer „doppelten Perspektive“ auf den transzendentalen Schematismus als „subsumtionsermöglichend“ und „erfahrungskonstituierend“. Bei Caimi (2015, 201 f.) heißt es lapidar, es gehe beim Schematismus darum, die „Subsumtion von konkreten Gegenständen [...] darzustellen. Es gilt also, die Kategorien als Erkenntnisbegriffe anzuwenden, sie als Prädikate den konkreten, einzelnen Erscheinungen beizulegen." - Bekanntlich hat Kants Präsentation des Schematismusproblems als das eines Subsumtionsverhältnisses, ob bei Bauch, Curtius oder Zschokke, Anlaß zu kritischen Analysen gegeben.
} 
sie geltungsdifferente, auf theoretische Geltung bezogene Setzung (durch den Subjektbegriff) und Bestimmung (durch den Prädikatbegriff) eines Gegenstandes ist. Entsprechend ergeben sich aus dieser erkenntnisfunktionalen Deutung des Urteils Prinzipien, die die Bestimmtheit jener Erkenntnisrelation und damit die Struktur des theoretischen Noemas (Gehalts) ausmachen. Sie machen dessen fundamentale Geltungsbestimmtheit aus und somit die grundlegenden geltungsfunktionalen Bestimmungsstücke des Verhältnisses von Denken (qua Gedanken) und Gegenstand, das die Erkenntnis ist. Das Denken ist hier also als Prinzip von Gegenständlichkeit thematisch (Wagner 1980c, 22 ff.; 1992, 227 ff.). Als Prinzip von Gegenständlichkeit tritt das Denken selbst (logisch) auseinander in das, was Gedanke (qua Gedanke von ...) und Gegenstand des Gedankens ist (Subjekt und Objekt, Bewußtsein und Welt o. ä.). Zwar indiziert im Falle empirischer Gegenständlichkeit das in den Sinnen Gegebene das Faktische, gleichwohl ist es das Denken, das die Sinnesgegebenheiten prüft und darüber entscheidet, was ,wirklich' (und nicht bloß vermeintlich) faktisch ist (1980c, § 21; 1992, 229 f., 232). Hinsichtlich der Geltung bleibt das Denken auf sich gestellt, ist es unbedingt, enthält es die Bedingungen der Geltung des Gedachten in sich. Auch für Wagner, wie für Rickert und Bauch, geht man daher von Anfang in die Irre, fängt man in der Bestimmung der Erkenntnis mit der „Getrenntheit zwischen den beiden Gliedern" Subjekt und Objekt, Bewußtsein und Welt u. dgl. an, mag streckenweise auch Kant diesem Irrtum verfallen sein (1992, 228; vgl. 1980c, 192), ist die Trennung doch das Werk des erkenntnisinteressierten Denkens: es bezieht sich als Prinzip von sich aus auf etwas von ihm Verschiedenes, sein Konkretum eben.

So wird auch bei Wagner sichtbar, $\mathrm{da}$ auf der Ursprungsebene der Erkenntnis kein radikaler Dualismus von Heterogenem vorliegt. Vielmehr ,konkretisiert" sich der Gedanke als Prinzip in Gedanken als Konkreta; er setzt und bestimmt den Gegenstand als das, was er an sich selbst ist (Wagner 1992, 230 f.). Freilich muß die objektive Gültigkeit mit Bezug auf den jeweiligen Gegenstand gewährleistet sein. Aber ebendies erfolgt dadurch, daß das konkrete Denken von Gegenständen, d. i. Erkennen, das Erkenntnis intendierende Denken, den „Prinzipien“ oder „Gesetzlichkeiten“ der Erkenntnis gehorcht.

Damit sind wir bei den geltungsnoematischen ,Stufen von Apriorität' angelangt. Denn die Erkenntnisprinzipien bilden kein homogenes Gefüge, sondern bestehen aus unterschiedlichen Typen mit ihrem jeweiligen Grad an Fundamentalität (Wagner 1980c, §§ 17, 19, 22, 23; 1980d; 1992, 235 ff.). Wagner unterscheidet näherhin vier Stufen.

Die primäre Konstitutivität bildet die 
schlechthin fundamentale Schicht der Erkenntnisprinzipien. Durch sie wird der Gedanke als Gedanke konstituiert: das Noema als Noema, das dann von einem Gegenstand allererst gelten kann. Wagners Lehrstück der primären Konstitutivität liefert die prinzipientheoretische Definition des Gedankens (Noemas) als eines solchen, und zwar in seiner Funktion, den Gegenstand bestimmen zu können (Gedanke von ...), in seiner erkenntnisfunktionalen Bestimmtheit also (1980c, 147 ff.; 1992, 237 f.). Wagner (1980c, 143) bezieht sich dabei im übrigen auf Rickert, der das Verhältnis von Form und Inhalt als Momente eines Ganzen habe „klären und zurechtrücken“ können. Sodann unterscheidet Wagner von der primären eine sekundäre Apriorität. Diese bildet den Inbegriff jener Prinzipien, die das Sein des Seienden (Seiende als Seiendes) ausmachen. Diesem Inbegriff reiner Seinsbegriffe und -grundsätze eignet anders als Wagners primärer Konstitutivität insofern nicht ein formal-, sondern einen inhaltlichkonstitutiver Charakter (1980c, § 19; 1992, 239 ff.). Über den Ansatz hinaus, den Wagners KonstitutivApriorisches für die Erkenntnis der Welt hat, stellt das Regulativ- und Systematisch-Apriorische sodann die Prinzipien dafür bereit, was die Erkenntnis als Mittel braucht, um ihre Bestimmungsintention $\mathrm{zu}$ vollbringen, d. i. den vollendeten Begriff des Seienden $\mathrm{zu}$ erreichen. Beim Regulativ-
Apriorischen handelt es sich um die methodologischen Prinzipien, um das Methodologisch-Apriorische: Prinzipien, die den Weg der Forschung bestimmen (1980c, § 22 f.; 1992, 241 ff.). Das Systematisch-Apriorische betrifft Prinzipien, die die jeweilige Erkenntnis auf das Gesamtsystem der Erkenntnis hin orientieren, von dem jede einzelne Erkenntnis immer auch geltungsmäßig abhängig ist (1980c, § 23; 1992, 242 f.). Damit ist der „innere Aufbau“ und die „Gliederung“ des Systems komplett (1980c, 211).

Kurzum: Die Geltungsstruktur der Erkenntnis wird auch bei Wagner über einen gestuften Inbegriff von geltungsnoematischen Prinzipien begrifflich erfaßt, der vom Ursprung bis in die Konkreta und deren Gesamtzusammenhang reicht. Auch Wagner gelangt zu diesem Inbegriff im Zuge einer geltungsnoematischen Reflexion auf den Anspruch der Erkenntnis, gültige Gegenstandsbestimmung zu sein, folglich durch eine immanente Explikation der Erkenntnisbeziehung. Eine radikale Heterogenität von Beziehungsgliedern, die der Vermittlung durch ein ,Drittes' bedürfte, gibt es daher auch bei Wagner nicht. So wie sämtliche Momente der sekundär-konstitutiven Apriorität nach einer identischen Regel unmittelbar mit denjenigen der primär-konstitutiven Apriorität zusammenhängen (Wagner 1980c, 175), so auch die darauf fundierten Stufen der Apriorität: sie alle ergeben sich 
aus einer wechselseitigen Implikation jeweiliger Beziehungsglieder (vgl. für das Regulative 1980c, 194). Entsprechend sind Wagner, wie er in bezug auf Kant formuliert, „Sinnlichkeit und Verstand" in transzendentaler Betrachtung keine zwei für sich selbst stehende Vermögen oder Funktionen: beide stehen in einem Verhältnis wechselseitiger Implikation (1980c, 192). ${ }^{43}$ (2) Bei Werner Flach, der mit sei- ner Erkenntnislehre die womöglich schärfste Fortbildung des dargelegten Fundierungsgedankens geliefert hat, ist es in der großen Linienführung (d. i. der geltungsnoematischen Begründung) nicht anders. 44 Freilich geht schon aus Flachs früher Prinzipienlehre der Anschauung hervor, daß die Problematik des Schematismus der Thematik der Vereinzelung der Erkenntnis zugehörig ist. Flach $(1963,146$ ff.) dis-

\footnotetext{
${ }^{43}$ Bunte $(2017,79$ ff.) versucht, Kant gegen Wagners Kritik am Ursprung der Kategorien aus den Urteilsformen zu verteidigen. Dabei nimmt er jedoch Bezug auf ein Theorem, dem bei Wagner eben aufgrund des von Bunte selbst dargestellten Primats der geltungsnoematischen Reflexion keine primär-fundierende Funktion zukommen kann: die Lehre von der „transzendentalen Apperzeption“. Bunte $(2017,79)$ spricht sogar von der Möglichkeit des Urteilens im Unterschied von der Einheit des Urteils; Wagner jedoch rückt das Urteil, nicht das Urteilen in den Vordergrund: die noetische Dimension des Erkenntnisvollzugs durch Erkenntnisakte hat einen nachgeordneten Rang. Ein ,Ich denke' und damit Kants apperzeptionstheoretische Pointierung der Objektivitätsbegründung lehnt Wagner als höchsten Punkt der Transzendentalphilosophie ausdrücklich ab; die Erkenntnisbeziehung selbst als Geltungsbeziehung ist ihm das Fundierende. Während Wagner den Übergang von der primären zur sekundären Konstitutivität in Kants metaphysischer Deduktion und dem dort dargelegten Verhältnis von Urteilsformen und Kategorien sieht, soll laut Bunte $(2017,83)$ Kants „transzendentales Schema“ den Übergang von der primären zur sekundären Apriorität leisten. Dies kann jedoch schon deshalb nicht der Fall sein, weil im Zuge von Kants transzendentaler Deduktion sich ergibt: „alle sinnlichen Anschauungen stehen unter den Kategorien“ (KrV B § 20). Der Übergang von der primären zur sekundären Apriorität ist also schon geleistet, und zwar begrifflich schon lange, weil Wagners sekundäre Apriorität noch indifferent ist bezüglich der Art des Gegebenseins (sinnlich, unsinnlich): es wird Gegenständlichkeit überhaupt (,Sein des Seienden') konstituiert (an der von Bunte angeführten Stelle, daß die Prinzipien der Gegenstandserkenntnis zugleich die der Erkenntnisgegenstände sein müssen (Wagner 1980c, 169), geht es nicht nur um die Relativierung dieser Konvertibilität durch die primäre Apriorität, sondern Wagner hat zuvor die für Kant „wesentliche“ Restriktion der Erkenntnis auf „Erfahrung“ verworfen (1980c, 168 f.)). Die sekundäre Apriorität kann also noch gar nicht unmittelbar die Dimension der „Selbstbestimmung des Denkens im Modus der Zeit“ (Bunte 2017, 84) betreffen. Überdies soll das Lehrstück vom transzendentalen Schema bei Kant nicht mehr dartun, daß Kategorien Gegenständlichkeit bestimmen, sondern wie sie auf Gegenstände der Erkenntnis angewendet werden können (KrV B 177, 167). Wagner hat Kants Schematismuslehre denn auch nicht für seine Bestimmung der sekundären Apriorität herangezogen, geschweige denn für den Übergang von der primären zur sekundären. Im Grunde geht es Bunte um die ursprüngliche Vermittlung von Anschauung und Begriff. Der Schematismus kann dazu insofern jedoch nichts beitragen, als er, jedenfalls in Kants Schematismuslehre der reinen Verstandesbegriffe, die Bezogenheit der Glieder, also den primären Stämmedualismus von Sinnlichkeit und Verstand bzw. Anschauung und Begriff, schon voraussetzt und daher allenfalls eine spätere Konstitutionsstufe ausmacht.

${ }^{44}$ Im Detail gibt es gravierende, der erkenntnisfunktionalen Fortbildung des Konkreszenzgedankens verdankte Differenzen. Sie sind systematisch höchst bedeutungsvoll. Es bleibt daher erstaunlich, daß, trotz der mittlerweile unüberschaubaren Anzahl an Bänden über Kants Aktualität, Flachs Erkenntnislehre, die durchgehend bemüht ist, Kants Aktualität herauszustellen, so wenig Aufmerksamkeit in der Kant-Forschung gefunden hat. Was nun die Differenzen zu Wagners Konzeption betrifft, ist es vor allem die geltungsfunktionale Kontinuität des Denkens bis zur Vereinzelung, die Flach zur Geltung bringt. Einen Dualismus von gegenstandsgebender Empfindung und gegenstandsbestimmender Kategorie gibt es insofern bei ihm nicht. Namentlich Wagners Unterscheidung einer sekundär-konstitutiven Apriorität, die die Prinzipien des Seienden enthält, in Unterscheidung von einer regulativen und systematischen Apriorität, die den Gang der Forschung reguliert und insofern einen heuristischen Charakter trägt, führt dazu, die sinnliche Gegebenheit als bloße Indikatorenfunktion der Konkretheit zu begreifen (heuristische Prinzipien haben strikte genommen mit der sinnlichen Bedingung der Kantischen Schematismuslehre nichts zu tun). Für Flach liegt darin eine konstitutionstheoretische ontologische Belastung der geltungsnoematischen Anlage der Kantischen Schematismuslehre, die sie geradezu um ihren Sinn bringt. Flachs Konzeption zufolge gehört Kants Lehre vielmehr zur Regionalisierung des Wissens, genauer: zur Thematik der empirischen Methodik. Konkretes Wissen, das empirisches Wissen ist, ist wie bei Kant Wissen von empirischer Gesetzlichkeit. Flach spezifiziert dieses Wissen näherhin als Beobachtungs-, Beschreibungs- und Erklärungswissen. Er modelt damit die Wagnersche Indizierungsfunktionalität des sinnlich Gegebenen zu einer Erprobungsfunktionalität. Kants Schematisierung wird entsprechend zur Flachschen Erprobung (der Sachadäquanz einer Sachaussage).
} 
kutiert sie in einem Kapitel über Anschauung und Urteil, in dem die Vereinzelung als urteilslogische Struktur erörtert wird. Ein Blick auf die diesbezüglichen Überlegungen ist schon deshalb hilfreich, weil in seiner späten Grundzügen der Erkenntnislehre der Schematismus nicht mehr als solcher behandelt wird: Offenbar ist er fundierungstheoretisch von nachgeordneter Relevanz; zudem, wie eben auch aus der Prinzipienlehre hervorgeht, gehört er zur Subjektivitätsproblematik, also zur Geltungsnoetik. Konkrete noematische Bestimmtheit ist auch für Flach nur als Vereinzelung der Geltung möglich. Sie qualifiziert sich nicht als Ursprungsstruktur, sondern als Anreicherung dieser Struktur zu ,thematisch gebundenem Sinn“, „Selbstvermittlung der Unendlichkeit zur Endlichkeit", „Subjektivität“ und damit zum ,inhaltlich erfüllten (kontingenten) Urteil“ (Flach 1963, 146 f.). Die Anschauung ist ihm dabei das Prinzip für die Art und Weise, wie sich die Geltung logisch vereinzelt. Entsprechend wird ihm die Sinnlichkeit zum prinzipientheoretischen Problem. In diesem Kontext heißt es, Kant habe im Schematismuskapitel eine Lösung versucht $(1963,147)$. Diese Lösung steht Flach zufolge jedoch unter der "Voraussetzung“ der Ungleichartigkeit von Kategorie und Erscheinung, so daß für Kant ein vermittelndes Drittes erforderlich ist (1963, 148). Eben weil Kant nach dem „Wie“ der Anwendung von Kategorien auf
Erscheinungen frage, gehe es ihm um eine „nähere Explikation“ der Struktur der transzendentalen Deduktion: das ,Dritte' sei das entscheidende Strukturmoment der Konkreszenzfunktion der Konstitution $(1963,148)$. Kant arbeitet gemäß Flachs Analyse also die konkreszierende Struktur noematischer Konstitution heraus. In Kants Lösung des Problems der Konkreszenz wird das Schema zu einer Regel, die dem Begriff sein Bild verschafft. Darin liegt auch für Flach, daß das Schema weniger ein vermittelndes Drittes ist, als vielmehr die eigentümliche Deduktionsstruktur der geltungslogischen Vereinzelung, die ebendeshalb von der Kategorie nicht losgelöst ist; vielmehr ist, wie Kant selbst schreibt, das Schema der „sinnliche Begriff eines Gegenstandes in Übereinstimmung mit der Kategorie" (1963, 149; vgl. KrV B 186). In Flachs Deutung des Schematismus überformt der Gedanke des Prinzips sachlich gesehen den eines vermittelnden Dritten. Das Schema realisiert einen konstitutiven Konkretionssinn; die ,Kategorien' werden konstitutionstheoretisch zu ,Grundsätzen'. Thematisch ist im Schematismuskapitel also die Konkreszenz der Erkenntnis. Sie vollzieht sich über die „formal-synthetische Struktur des Urteils“, also geltungsnoematisch. Sie führt, wie in Rickerts oder Wagners Geltungsnoematik etwa, zu einer Klärung der Prädikation im Urteil. Im Urteil wird die reine Heterogenität der Ursprungsphäre überwunden: es 
kommt zur Heterogenität in der Homogenität (1963, 150 f.). Wie es heißt, ist mit der Prädikation das „urteilslogische Äquivalent" zur Vereinzelungsthematik der Subjektstheorie (als Lehre des Erkennens) gefunden (1963, 156).

In seiner Erkenntnislehre hat Flach dies alles prinzipientheoretisch wesentlich differenzierter ausgeführt. Kants Schematismuslehre kommt dabei allerdings nur am Rande zur Sprache. Es bestätigt sich freilich der bislang herausgearbeitete Leitfaden: Kants Schematismus hat seine Stelle in Flachs "Methodenlehre" (1994, Kap. 4), genauer: er wird thematisch, sobald die „speziellen Methoden“ behandelt werden, d. h. die „Regionalisation des wissenschaftlichen Wissens" (1994, Kap. 4.4). Flachs Logik handelt in geltungsreflexiver Weise - in "formalnoematischer Reflexion“ - von jener Aprioritätsdimension, die die Struktur der Erkenntnis, deren prinzipielle Bestimmtheit, ausmacht: der "geltungsnoematischen Struktur“, der „logischen Form des Noema", der Struktur des Urteils (1994, Kap. 3, zit. 249). Während die Logik die „Konstitutivität der geltungsnoematischen Struktur“ (1994, 249) diskutiert, geht es der Methodenlehre nicht um die Konstitution der Erkenntnis, sondern um die mit der konstitutiven Struktur der Erkenntnis komplementär verbundene, auf der konstitutiven Formalität als ihrem Geltungsboden ruhende „Organisation der Erkenntnis“ (1994, Kap. 4, zit. 355): das
Methodologische betrifft die Dimension der „regulativen Apriorität“ (1994, Kap. 4.1.2); sie betrifft näherhin den sich selbst determinierenden „Prozeß-, Progreß-, Differentiations- und Integrationscharakter" der Erkenntnis (1994, Kap. 4.1.1). Dank seiner Methodizität wird das Wissen zur konkreten Erkenntnis: die regulativ-apriorischen Bedingungen machen die Erkenntnis zur „sachbezogenen und sacherfüllten“ Bestimmung (1994, 394). Der Methodizität der Erkenntnis eignen dabei zwei Dimensionen (Regulationsebenen): eine universale und eine spezielle Dimension. Es gibt also universelle, die Erkenntnis in genere regulierende und damit die Einheit des wissenschaftlichen Wissens ausmachende Methoden und spezielle, die Erkenntnis in specie regulierende und damit die Regionalisation des wissenschaftlichen Wissen ausmachende Methoden (1994, 398, Kap. 4.3. und 4.4). Durch die ersteren ist Erkenntnis überhaupt Sachbestimmung (konkrete Erkenntnis), durch die letzteren ist Erkenntnis diese oder jene Sachbestimmung: zusammen prinzipiieren sie die Erkenntnis als konkrete Erkenntnis.

Auf der Regulationsebene der speziellen Methoden äußert Flach sich zu Kants Schematismus reiner Verstandesbegriffe. Durch die spezifische Regulativität besondert sich nämlich die in der universalen Regulativität konzipierte Theoriebestimmtheit überhaupt der Erkenntnis. Als besondere Theo- 
rie ist sie nicht nur durch Geltungsapodiktizität, sondern auch durch Geltungskontingenz gekennzeichnet. Das Prinzip der Geltungskontingenz aber wird durch den Begriff der Anschauung verbürgt. Die Theoriebestimmtheit besonderer Theorie ist also als anschauungsdifferente Bestimmtheit zu diskutieren (Flach 1994, 562). So kommt es in Flachs Methodologie zur Unterscheidung theoretischer Anschauungsmodi, näherhin der reinen und der empirischen Anschauung (1994, 563, vgl. Kap 4.4.1 u. 4.4.2). Der Modus empirischer Anschauung spezifiziert dabei die von den universalen Methoden prinzipiierte Theoriebestimmtheit überhaupt der Erkenntnis zur Bestimmtheit empirischer Theorie $(1994,577)$. Diese kennt nur Sachaussagen, deren Sachadäquanz der Erprobung unterliegt; die Erprobung bildet für Flach den „methodischen Brennpunkt der Erfahrungserkenntnis“: empirische Erkenntnis ist primär in der Perspektive der Erprobung zu verstehen $(1994,578)$. Flach führt im Anschluß an, aber auch in Abhebung von Kant (1994, 587 ff.) hier den Begriff der Wahrnehmung als einer methodologischen Geltungsqualifikation ein (1994, 579 ff.). Die Wahrnehmung ist der methodischen Bestimmtheit der Sa- chaussage integriert: sie ist methodische Bedingung von Erfahrungserkenntnis, kein Habitus (mehr), sondern eine Erkenntnisfunktion (Flach 1994, 580 f.). 45 Die methodische Beugung (Formierung) der Wahrnehmung gestaltet sich hinsichtlich der Erprobung der Sachadäquanz der Erfahrungserkenntnis als einen Funktionsverlauf, der sich von der minimalen zur maximalen methodischen Beugung erstreckt: von der Beobachtung zur Beschreibung und schließlich zur Erklärung (1994, 587 f.; Kap. 4.4.2.1 ff.). So wird die Sachadäquanz der Sachaussage gesichert und folglich die Sache als Tatsache wissenschaftlich gefaßt. Wie angedeutet, Flach beruft sich dabei systematisch durchaus auf Kant, näherhin auf Kants Lehre vom Wahrnehmungsurteil 4 die im Zusammenhang mit der Aprioritätsproblematik den für Flach wichtigen Begriff der empirischen Signifikanz hergibt: die Wahrnehmung erhält eine regulativapriorische Prinzipienfunktion (1994, 589). Durch die methodische Beugung wird die Wahrnehmung zur Erkenntniskraft.

Die anfängliche methodische Beugung der Wahrnehmung durch die Beobachtung wird von Flach als "Schematisierung“ der Kategorie der „Realität“ aufgefaßt: die Kategorie der Re-

\footnotetext{
${ }^{45}$ Damit bindet Flach zugleich das erkennende Subjekt als sinnliches Vernunftwesen, d. i. als empirisches (,tatsächliches“) Subjekt, in die Erkenntnisbestimmung ein: es nutzt seine sinnliche Ausstattung für die Erkenntnis (1994, 581 ff.). Es ist für Flach so das „regulative Gegenstück zu dem konstitutiven Derivat des Subjekts als Bewußtsein“ $(1994,582)$.

${ }^{46}$ Gegenüber allen nachkantischen Bemühungen, des Problems der empirischen Signifikanz Herr zu werden, behalte sie eine „inspirative Überlegenheit" (Flach 1994, 596).
} 
alität gilt Flach als die für jede Wahrnehmung fundamentale Sachvoraussetzung (1994, 597). Die Beschreibung setzt die methodische Beugung dahingehend fort, daß sie die mit der Kategorie der Realität durchsetzte Wahrnehmung mit weiteren Kategorien durchsetzt: es ergibt sich eine vielfältige "Schematisierung", die zur Sachvorstellung der Wahrnehmung führt (1994, 619). Es findet eine „Durchdringung" des von der Beobachtung bereitgestellten konstatierten Datums statt: die „monokategoriale Realitätsbehauptung" wird zu einem "polykategorialen Netzwerk" der Sachbestimmung, näherhin der Beschreibung (Deskription) $(1994,620)$. Sie wird schließlich der Erklärung zugeführt.

An diesem Sachverhalt der methodischen Beugung der Wahrnehmung durch Beobachtung, Beschreibung und Erklärung ist für meine These von besonderem Interesse, daß Flach in seiner Empiriologie der Beschreibung auf Kants Schematismus eingeht (1994, 626 ff.). Er unterstreicht gegen empiristische Ansätze, daß Kant die transzendentale Deduktion der erfahrungskonstituierenden Begriffe bis zum Aufweis der Bedingungen führt, die zum deskriptiven Erfahrungsurteil führen: Bedingungen, die den Verstandesbegriff das Material des wahrnehmungsmäßig Gegebenen zu dessen „Bild“ gestalten lassen $(1994,626)$, wie Flach offenbar im Anschluß an Kants Schematismuskapitel formuliert. Das Schema ist der Leitfaden der bildlichen Realisierung des Verstandesbegriffs; er richtet die gegenstandskonstituierende Synthesis auf die für Menschen spezifische $\mathrm{Ge}$ gebenheitsweise aus: auf die Sinnlichkeit; die Synthesis wird zur "figürlichen" Synthesis, zur Erzeugung einer begrifflichen Figur. Sie wird Flach zur Synthesis, der die „objektive Realität“ zur „Konkretion“ wird (1994, 626). Kants Subsumtion der Erscheinungen unter Verstandesbegriffe, letztlich unter die reinen, bringt Flachs Erkenntnislehre zufolge die methodische Bestimmtheit der Beschreibung zum Ausdruck. Auf der Ebene der Erscheinung ist die anschauliche Bestimmtheit zur begrifflichen Bestimmtheit gebracht (1994, 626 f.).

Im Ergebnis ist die Heterogenitätsthese Kants auch bei Flach gänzlich in die Entfaltung der internen Bezüge der geltungsnoematischen Struktur zurückgenommen. Da gibt es nur den Weg von ihr als Ursprung aller Bestimmtheit zum bestimmten Konkretum. Die Schematisierungsleistung der Spontaneität des Denkens wird festgehalten, Kants apperzeptionstheoretische Pointierung und die mit ihr einhergehende Äußerlichkeit von Gegebenem und Bestimmung des Gegebenen in einer geltungsnoematischen Konstitutions- und Regulationsordnung aufgehoben. Der positive Sinn von Kants Schematismuslehre erweist sich, ob bei den diskutierten Neukantianern oder den Transzendentalphilosophen der Nachkriegszeit, als - Methodologie. 


\section{Literaturverzeichnis}

ALLISON, Henry E. Kant's Transcendental Idealism. Rev. and enl. New Haven: Yale University Press, 2004.

BAUCH, Bruno. Über den Begriff des Naturgesetzes. Kant-Studien 19: 303-37, 1914.

. Idealismus und Realismus in der Sphäre des philosophischen Kritizismus: Ein Verständigungsversuch. KantStudien 20: 97-116, 1915.

Das transzendentale Subjekt: Eine transzendentalphilosophische Skizze. Logos 12: 23-49, 1923/24.

Immanuel Kant. 3. Aufl. Berlin, Leipzig: de Gruyter, 1923a

Wahrheit, Wert und Wirklichkeit. Leipzig: Meiner, 1923b.

Die Idee. Leipzig: Meiner, 1926.

Theoretische Philosophie (1931). In Neukantianismus: Texte der Marburger und der Südwestdeutschen Schule, ihrer

Vorläufer und Kritiker, hg. v. H.-L Ollig, 243-78. Darmstadt: Wissenschaftliche Buchgesellschaft, 1982.

BIRRER, Mathias. Kant und die Heterogenität der Erkenntnisquellen. Berlin, Boston: de Gruyter, 2017.

BRANDOM, Robert. Making it Explicit: Reasoning, Representing, and Discursive Commitment. Cambridge et al: Harvard University Press, 1994.

BUNTE, Martin. Erkenntnis und Funktion: Zur Vollständigkeit der Urteilstafel und Einheit des kantischen Systems. Berlin, Boston: de Gruyter, 2016.

. Wissen und Bestimmtheit: Primär- und sekundärkonstitutive Apriorität bei Kant und Hans Wagner In Reflexion und konkrete Subjektivität: Beiträge zum 100. Geburtstag von Hans Wagner (1917-2000), hg. v. Christian Krijnen und Kurt W. Zeidler, 75-89. Wien: Ferstl Perz, 2017.

CAIMI, Mario. Der Schematismus der reinen Verstandesbegriffe. In Kants Theorie der Erfahrung, hg. v. Rainer Enskat, 201-37. Berlin, München, Boston: de Gruyter, 2015.

CASSIRER, Ernst.Das Erkenntnisproblem in der Philosophie und Wissenschaft der neueren Zeit. Teil 2. Nachdr. d. 3. Darmstadt: Wissenschaftliche Buchgesellschaft, 1994a.

. Kants Leben und Lehre. Nachdr. der Ausg. New Haven. Darmstadt: Wissenschaftliche Buchgesellschaft, 1994b.

COHEN, Hermann. Kants Begründung der Aesthetik. Berlin: Dümmler, 1889. . Logik der reinen Erkenntnis. Berlin: Cassirer, 1902.

Kants Begründung der Ethik: Nebst ihren Anwendungen auf Recht, Religion und Geschichte. 2. Aufl. Berlin: Cassirer, 1910.

Kants Theorie der Erfahrung. 3. Aufl. Berlin: Cassirer, 1918.

COHN, Jonas. Voraussetzungen und Ziele des Erkennens: Untersuchungen über die Grundfragen der Logik. Leipzig: Engelmann, 1908.

. Selbstdarstellung In Die Philosophie der Gegenwart in Selbstdarstellungen, hg. v. Raymund Schmidt. 2. verb., 1-21. Leipzig: Meiner, 1923a.

Theorie der Dialektik: Formenlehre der Philosophie. Leipzig: Meiner, $1923 b$.

Wertwissenschaft. Stuttgart: Fromanns, 1932.

CURTIUS, Ernst R. Das Schematismusproblem in Kants Kritik der reinen Vernunft: Eine philologische Untersuchung. Kant-Studien 19: 338-66, 1914.

DÜSING, Klaus. Schema und Einbildungskraft in Kants Kritik der reinen Vernunft. In Aufklärung und Skepsis: Studien zur Philosophie und Geistesgeschichte des 17. und 18. Jahrhunderts, hg. v. Lothar Kreimendahl, 47-71. Stuttgart-Bad Cannstatt: Frommann-Holzboog, 1995.

FLACH, Werner. Zur Prinzipienlehre der Anschauung: Bd. 1: Das spekulative Grundproblem der Vereinzelung. Hamburg: Meiner, 1963.

. Grundzüge der Erkenntnislehre: Erkenntniskritik, Logik, Methodologie. Würzburg: Königshausen Neumann, 1994.

Kants Lehre von der Gesetzmäßigkeit der Empirie: Zur Argumentation der Kantischen Schematismuslehre. Kant-Studien 92: 464-73, 2001.

Die Idee der Transzendentalphilosophie: Immanuel Kant. Würzburg: Königshausen Neumann, 2002.

. Die kantische Hypothek. In Kant zu Geschichte, Kultur und Recht, hg. v. Wolfgang Bock, 15-25. Berlin: Duncker Humblot, 2015.

GONZÁLEZ PORTA, Mario A. Transzendentaler "Objektivismus". Bruno Bauchs kritische Verarbeitung des Themas der Subjektivität und ihre Stellung innerhalb der Neukantianischen Bewegung. Frankfurt am Main: Peter Lang, 1990.

HEGEL, Georg W. F. Werke in zwanzig Bänden. Hg. v. Eva Moldenhauer und Karl M. Michel. Frankfurt am Main: Suhrkamp, 1971.

HEIDEGGER, M. Die Frage nach dem Ding. Zu Kants Lehre von den transzendentalen Grundsätzen. Tübingen: Niemeyer, 1962.

. Kant und das Problem der Metaphysik (1929). In Gesamtausgabe. Frankfurt am Main: Klostermann, 1991. 
HEIDEMANN, Dietmar. Anschauung und Begriff: Ein Begründungsversuch des Stämme-Dualismus in Kants Erkenntnistheorie. In Aufklärungen (FS K. Düsing), hg. v. Kristina Engelhard, 65-90. Berlin: Duncker Humblot, 2002.

HEINZ, Marion; KRIJNEN, Christian. Kant im Neukantianismus: Fortschritt oder Rückschritt? Würzburg: Königshausen und Neumann, 2007.

HÖFFE, Otfried. Kants Kritik der reinen Vernunft: Die Grundlegung der modernen Philosophie. München: Beck, 2003.

KANT, Immanuel. Kants gesammelte Schriften. Bd. I-XXIX, hrsg. v. Königlich-Preußische Akademie der Wissenschaften et al., Berlin: De Gruyter (zitiert als: AA), 1910.

KEMP SMITH, Norman. A Commentary on Kant's 'Critique of Pure Reason'. London: Macmillan, 1918.

KRIJNEN, Christian. Nachmetaphysischer Sinn: Eine problemgeschichtliche und systematische Studie zu den Prinzipien der Wertphilosophie Heinrich Rickerts. Würzburg: Königshausen Neumann, 2001.

. Das konstitutionstheoretische Problem der transzendentalen Ästhetik in Kants ,Kritik der reinen Vernunft' und seine Aufnahme im südwestdeutschen Neukantianismus. In Kant im Neukantianismus: Fortschritt oder Rückschritt? hg. v. Marion Heinz und Christian Krijnen, 109-34. Würzburg: Königshausen und Neumann, 2007.

. Kants Subjektstheorie und die Grundlegung einer philosophischen Anthropologie. Zeitschrift für philosophische Forschung 62: 254-73, 2008a.

Philosophie als System: Prinzipientheoretische Untersuchungen zum Systemgedanken bei Hegel, im Neukantianismus und in der Gegenwartsphilosophie. Würzburg: Königshausen Neumann, 2008b.

- Geschichtsphilosophie bei Kant, im Neukantianismus und im gegenwärtigen Kantianismus In Der Begriff der Geschichte im Marburger und südwestdeutschen Neukantianismus, hg. v. Christian Krijnen und Marc de Launay, 29-57. Würzburg: Königshausen Neumann, 2013.

. Gegenstandskonstitution bei Husserl und in der klassischen deutschen Philosophie: Eine problemgeschichtliche Deutungslinie In Husserl und die klassische deutsche Philosophie: Husserl and Classical German Philosophy, hg. v. Faustino Fabbianelli und Sebastian Luft, 115-31. Cham, Heidelberg, New York, Dordrecht, London: Springer, 2014a.

. Transzendentaler Idealismus und empirischer Realismus In Wissenschaftsphilosophie im Neukantianismus: Ansätze

- Kontroversen - Wirkungen, hg. v. Christian Krijnen und Kurt W. Zeidler, 11-56. Würzburg: Königshausen Neumann, 2014b.

KRIJNEN, Christian.; ZEILDER, Kurt. Gegenstandsbestimmung und Selbstgestaltung: Transzendentalphilosophie im Anschluss an Werner Flach. Würzburg: Königshausen Neumann, 2011.

. Reflexion und konkrete Subjektivität: Beiträge zum 100. Geburtstag von Hans Wagner (1917-2000). Wien: Ferstl Perz, 2017.

LASK, Emil. Die Logik der Philosophie und die Kategorienlehre In Gesammelte Schriften Bd. II, hg. v. Eugen Herrigel, 1-182. Tübingen: Mohr, 1911.

. Die Lehre vom Urteil In Gesammelte Schriften, Bd. II, hg. v. E. Herrigel, 283-463. Tübingen: Mohr, 1912.

NACHTSHEIM, Stephan. Emil Lasks Grundlehre. Tübingen: Mohr, 1992.

. La "struttura formale": Un' osservazione sul concetto di fondazione ultima In Emil Lask: Un secolo dopo, hg. v. Stefano Besoli und Roberto Redaelli, 11-22. Macerata: Quodlibet, 2017.

NATORP, Paul. Kant und die Marburger Schule. Kant-Studien 17: 193-221, 1912.

. Zum Gedächtnis Kants (1904) In Immanuel Kant zu ehren, hg. v. Joachim Kopper und Rudolf Malter, $236-60$. Frankfurt am Main: Suhrkamp, 1974.

PRINGE, Hernán. Bruno Bauch and the Comprehensibility of Nature. Kriterion 56: 355-70, 2015.

RICKERT, Heinrich. Fichtes Atheismusstreit und die Kantische Philosophie. Berlin: Reuther Reichard, 1889. . Zwei Wege der Erkenntnistheorie: Transcendentalpsychologie und Transcendentallogik. Kant-Studien 14: 169-228, 1909.

Urteil und Urteilen. Logos 3: 230-45, 1912.

System der Philosophie: Erster Teil: Allgemeine Grundlegung der Philosophie. Tübingen: Mohr, 1921.

Alois Riehl. Logos 13: 162-85, 1924/25.

Das Eine, die Einheit und die Eins: Bemerkungen zur Logik des Zahlbegriffs. 2. umg. Aufl. Tübingen: Mohr, 1924.

Kulturwissenschaft und Naturwissenschaft. 6. u. 7. durchg. u. erg. Aufl. Tübingen: Mohr, 1926.

. Der Gegenstand der Erkenntnis: Einführung in die Transzendentalphilosophie. 6. verb. Aufl. Tübingen: Mohr, 1928.

Die Grenzen der naturwissenschaftlichen Begriffsbildung: Eine logische Einleitung in die historischen Wissenschaften.

5. verb. u. erw. Aufl. Tübingen: Mohr, 1929.

Die Logik des Prädikats und das Problem der Ontologie. Heidelberg: Winter, 1930.

Grundprobleme der Philosophie: Methodologie, Ontologie, Anthropologie. Tübingen: Mohr, 1934.

Die Erkenntnis der intelligiblen Welt und das Problem der Metaphysik: Erster Teil (1927) In Unmittelbarkeit und

Sinndeutung: Aufsätze zur Ausgestaltung des Systems der Philosophie, hg. v. August Faust, 97-138. Tübingen: Mohr, 1939a. 
Die Erkenntnis der intelligiblen Welt und das Problem der Metaphysik: Zweiter Teil (1929). In Unmittelbarkeit und Sinndeutung: Aufsätze zur Ausgestaltung des Systems der Philosophie, hg. v. August Faust, 139-85. Tübingen: Mohr, 1939b.

. Unmittelbarkeit und Sinndeutung: Aufsätze zur Ausgestaltung des Systems der Philosophie. Hg. v. August Faust. Tübingen: Mohr, 1939c.

WAGNER, Hans. Der Argumentationsgang in Kants Deduktion der Kategorien. Kant-Studien 71: 352-66, 1980a.

Kritische Betrachtungen zu Husserls Nachlass (1953). In Kritische Philosophie: Systematische und historische Abhandlungen, hg. v. Karl Bärthlein und Werner Flach, 362-96. Würzburg: Königshausen Neumann, 1980 b. Philosophie und Reflexion. 3. Aufl. München, Basel: Reinhardt, 1980c.

Über den Begriff des Idealismus und die Stufen der theoretischen Apriorität (1952). In Kritische Philosophie: Systematische und historische Abhandlungen, hg. v. Karl Bärthlein und Werner Flach, 28-41. Würzburg: Königshausen Neumann, 1980d.

. Die Würde des Menschen: Wesen und Normfunktion. Würzburg: Königshausen Neumann, 1992.

WINDELBAND, Wilhelm. Vorwort (1883). In Präludien: Aufsätze und Reden zur Philosophie und ihrer Geschichte. 5. Aufl. 2 Bd., III-IV. Tübingen: Mohr, 1915.

ZOCHER, Rudolf. Kants Transzendentale Deduktion der Kategorien. Zeitschrift für philosophische Forschung 8: 161-194, 1954.

. Kants Grundlehre. Erlangen: Universitätsbund, 1959.

Recebido: $15 / 07 / 2020$

Aprovado: $15 / 11 / 2020$

Publicado: $30 / 12 / 2020$ 
